\title{
Probing the CP properties of top Yukawa coupling at an $e^{+} e^{-}$collider
}

\author{
Kaoru Hagiwara, ${ }^{a, b}$ Hiroshi Yokoya ${ }^{c}$ and Ya-Juan Zheng ${ }^{d}$ \\ ${ }^{a}$ KEK Theory Center and Sokendai, \\ Tsukuba, Ibaraki 305-0801, Japan \\ ${ }^{b}$ Centre for Cosmology, Particle Physics and Phenomenology (CP3), \\ Université Catholique de Louvain, \\ B-1348 Louvain-la-Neuve, Belgium \\ ${ }^{c}$ Quantum Universe Center, KIAS, \\ Seoul 02455, Korea \\ ${ }^{d}$ Department of Physics, Osaka University, \\ Osaka 560-0043, Japan \\ E-mail: kaoru.hagiwara@kek.jp, hyokoya@kias.re.kr, \\ yjzheng@het.phys.sci.osaka-u.ac.jp
}

ABSTRACT: We study consequences of CP violation in the $h t \bar{t}$ Yukawa coupling through the process $e^{+} e^{-} \rightarrow h(125) t \bar{t}$. The helicity amplitudes are calculated in the $t \bar{t}$ rest frame, where the initial $e^{+} e^{-}$current and the final Higgs boson have the same three-momentum. CP-violating asymmetries appear not only in the azimuthal angle between the $e^{+} e^{-}$plane and the $t \bar{t}$ plane about the Higgs momentum direction, but also in the correlated decay angular distributions of $t$ and $\bar{t}$. Complete description of the production and decay angular distributions are obtained analytically, including both leptonic and hadronic decays of $t$ and $\bar{t}$. We study the ultimate sensitivity to the CP-violating $h t \bar{t}$ coupling at a few center-of-mass energies. Our analysis shows that the possibility of discovering CP-violating $h t \bar{t}$ coupling improves significantly by studying $t \bar{t}$ decay angular correlations, and more importantly, by increasing its energy upgrade target from $\sqrt{s}=500 \mathrm{GeV}$ to $550 \mathrm{GeV}$.

Keywords: CP violation, Higgs Physics, Beyond Standard Model

ARXIV EPRINT: 1712.09953 


\section{Contents}

1 Introduction 1

2 Helicity amplitudes and density matrix 3

3 Cross sections and QCD corrections 13

$\begin{array}{lll}3.1 & \text { Leading-order production cross section } & 13\end{array}$

$\begin{array}{lll}3.2 & \text { QCD corrections } & 13\end{array}$

4 Sensitivity of $e^{+} e^{-} \rightarrow h t \bar{t}$ experiments on $\xi_{h t t} \quad 17$

$\begin{array}{ll}4.1 \text { Total cross section } & 17\end{array}$

4.2 Differential cross sections 20

4.3 Results 22

$\begin{array}{lll}5 & \text { Summary and discussions } & 27\end{array}$

$\begin{array}{ll}\text { A Top and antitop quark decay density matrix } & 28\end{array}$

B HELAS phase convention for the density matrix 33

\section{Introduction}

All the current measurements of the Higgs boson $h(125)$ are consistent with predictions of the Standard Model (SM) of particle physics [1, 2]. Detailed studies of all its properties should be of at most importance in our search for the physics of the symmetry breakdown, or the origin of the SM.

In this paper, we show how the top quark Yukawa coupling, the $h t \bar{t}$ coupling, can be studied in the process $e^{+} e^{-} \rightarrow h t \bar{t}$ at linear colliders such as ILC and CLIC. We develop techniques that allow us to perform the optimal measurements of the $h t \bar{t}$ coupling in a clean $e^{+} e^{-}$collider environment. In order to quantify the impacts of our proposal to measure full production and decay angular correlations of both semi-leptonic and hadronic decays of top and anti-top quarks, we adopt the following simple effective Lagrangian for the $h t \bar{t}$ coupling

$$
\mathcal{L}=-g_{h t t} h \bar{t}\left[\cos \xi_{h t t}+i \sin \xi_{h t t} \gamma_{5}\right] t
$$

with two real parameters, $g_{h t t}$ and $\xi_{h t t}$. The effective Lagrangian eq. (1.1) reduces to the SM $h t \bar{t}$ coupling when

$$
g_{h t t}=g_{h t t}^{\mathrm{SM}}=\frac{m_{t}}{v}, \quad \xi_{h t t}=0
$$


with the top quark mass $m_{t}$ and the vacuum expectation value (VEV) $v \simeq 246 \mathrm{GeV}$ of the SM Higgs field. Nonzero values of $\sin \xi_{h t t}$ term in eq. (1.1) implies CP violation, while the magnitude of the $g_{h t t}$ is measured with respect to its SM value

$$
\kappa_{h t t}=g_{h t t} / g_{h t t}^{\mathrm{SM}}
$$

The sign of $\cos \xi_{h t t}$ term, or that of $g_{h t t} \cos \xi_{h t t}$, is measured with respect to the sign of the $h Z Z$ coupling,

$$
g_{h Z Z}=\kappa_{h Z Z} g_{h Z Z}^{\mathrm{SM}}=\kappa_{h Z Z} \frac{m_{Z}^{2}}{v},
$$

because the amplitudes with the $h t \bar{t}$ couplings and those with the $h Z Z$ coupling interfere in the process $e^{+} e^{-} \rightarrow h t \bar{t}$. Therefore, the amplitudes depend on the three parameters,

$$
\kappa_{h t t}, \kappa_{h Z Z}>0, \quad-\pi \leq \xi_{h t t}<\pi
$$

It is worth noting that by the time an $e^{+} e^{-}$collider starts studying the $h t \bar{t}$ production process, we should have constraints on $\left(\kappa_{h t t}, \xi_{h t t}, \kappa_{h Z Z}\right)$ from the LHC: $\kappa_{h Z Z}$ can be measured from $\Gamma\left(h \rightarrow Z Z^{*}\right)$ and from the weak boson fusion production cross section; $\left(\kappa_{h t t}, \xi_{h t t}\right)$ are measured from $h t \bar{t}$ production process [3-8]; and $\left(\kappa_{h t t}, \xi_{h t t}, \kappa_{h Z Z}\right)$ can be constrained in the single top plus Higgs production process [4, 9-11]. Some CP violating asymmetries including top decay lepton distributions are studied in [4]. The couplings are constrained by the perturbative unitarity $[12,13]$, and affect loop induced amplitudes for $\Gamma(h \rightarrow g g)$ and $\Gamma(h \rightarrow \gamma \gamma)$. The role of the $e^{+} e^{-}$collider experiments should be their refinements and possible discovery of non-SM physics in the $h t \bar{t}$ coupling, such as CP violation. Differential cross sections and top polarizations are studied in $[14,15]$, including $\mathrm{CP}$ violating observables [14]. Ref. [16] studied asymmetries in decay lepton angular correlations in Higgs plus topponium production at $\sqrt{s}=500 \mathrm{GeV}$.

At present, only the $h Z Z$ coupling strength $\kappa_{h Z Z}$ has been measured to be

$$
\kappa_{h Z Z} \gtrsim 0.85
$$

more or less free from detailed model assumptions [2]. We expect the measurement to improve significantly by the time of an $e^{+} e^{-}$collider experiment, and we set

$$
\kappa_{h Z Z}=1
$$

throughout our analysis. All the results are insensitive to this assumption, which does not change significantly by varying $\kappa_{h Z Z}$ in the $0.85<\kappa_{h Z Z} \leq 1$ range, because the amplitudes with the $h Z Z$ coupling are subleading in $e^{+} e^{-} \rightarrow h t \bar{t}$ process. We allow the two real parameters $\left(\kappa_{h t t}, \xi_{h t t}\right)$ to vary freely in our analysis, with the understanding that they should be constrained significantly by the LHC experiments when the $e^{+} e^{-}$collider experiments are performed.

Before starting our studies, we find it instructive to examine a very specific limit of CP violation in the Higgs couplings where the sole origin of $\mathrm{CP}$ violation in the Higgs sector is 
in the Higgs potential or the Higgs self interactions, while all the other couplings including Yukawa couplings are CP conserving. It is like a milli-weak theory of $\mathrm{CP}$ violation for the neutral $K$ system [17], where CP violation is confined to the $K^{0}-\bar{K}^{0}$ mixing. The scenario can be realized in any multi-doublet models, where the observed Higgs boson, $h(125)$, is a linear combination

$$
h(125)=O_{h H} H+O_{h H^{\prime}} H^{\prime}+O_{h A} A,
$$

where $H$ and $H^{\prime}$ are CP-even while $A$ is a CP-odd neutral components of the Higgs boson. If all the Yukawa interactions of the current states, $H, H^{\prime}$ and $A$ are $\mathrm{CP}$ conserving, the sole origin of all the CP-violating couplings of $h(125)$ is the mixing matrix element $O_{h A}$. We can choose $H$ to be the fluctuation of the full SM Higgs VEV that gives the weak boson masses, and the orthogonal states $H^{\prime}$ and $A$ are fixed uniquely in two Higgs doublet models (2HDM). Specific form of the $h t \bar{t}$ couplings is model dependent. For instance in type II $2 \mathrm{HDM}$, the $h Z Z$ and $h t \bar{t}$ couplings are

$$
\begin{aligned}
\kappa_{h Z Z} & =O_{h H} \\
\kappa_{h t t} \cos \xi_{h t t} & =O_{h H}+O_{h H^{\prime}} \frac{1}{\tan \beta} \\
\kappa_{h t t} \sin \xi_{h t t} & =O_{h A} \frac{1}{\tan \beta}
\end{aligned}
$$

where $\tan \beta$ is the ratio of the two VEV's. It is clear that in this scenario, $\kappa_{h Z Z}=1$ implies $O_{h H^{\prime}}=O_{h A}=0$ from the orthogonality of the mixing matrix. Even in this scenario, significant $\mathrm{CP}$ violation is possible for the $h t \bar{t}$ coupling for small $\tan \beta$, if $\kappa_{h Z Z} \sim 0.9$.

The paper is organized as follows. In section 2 , we calculate the helicity amplitudes of the $e^{+} e^{-} \rightarrow h t \bar{t}$ process in the $t \bar{t}$ rest frame. In section 3, we show the numerical results of production cross section, invariant mass distribution of the $h t \bar{t}$ production process with next-to-leading-order (NLO) QCD corrections including Coulomb resummation for topponium formation. In section 4 , we introduce the density matrix formalism to express full kinematical distributions including $t$ and $\bar{t}$ decay angular correlations, including both semi-leptonic $(t \rightarrow b \bar{\ell} \nu)$ and hadronic $(t \rightarrow b \bar{d} u)$ decays. By accounting for uncertainties in quark jet-flavor identification, we study how the sensitivity to $\mathrm{CP}$ violation increases by measuring full distributions, at $\sqrt{s}=500 \mathrm{GeV}, 550 \mathrm{GeV}$ and $1000 \mathrm{GeV}$. Summary and conclusions are given in section 5. In appendix A, we show $t$ and $\bar{t}$ decay density matrices for both semi-leptonic and hadronic decays. In appendix B, we review HELAS phase convention that affects our production density matrix elements.

\section{Helicity amplitudes and density matrix}

As shown in figure 1, three Feynman diagrams contribute to the process $e \bar{e} \rightarrow h t \bar{t}$. The first two are with the $h t \bar{t}$ coupling in eq. (1.1), and the third is with the $h Z Z$ coupling for which we assume the SM value as in eq. (1.7) in this report.

We calculate the helicity amplitudes of the process in the $t \bar{t}$ rest frame, where the initial $e^{+} e^{-}$current and the produced Higgs boson have the same three-momentum. We 


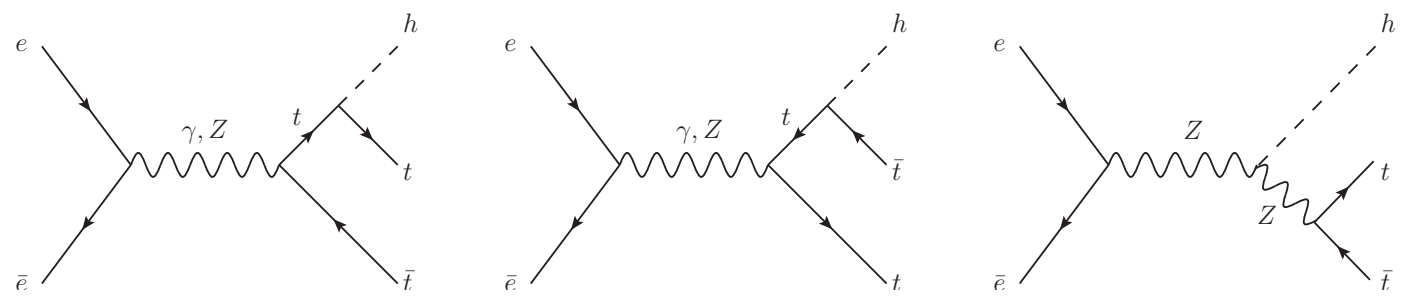

Figure 1. The Feynman diagrams of the $e \bar{e} \rightarrow h t \bar{t}$ process.

denote the helicity amplitudes as

$$
\mathcal{M}_{\alpha \sigma \bar{\sigma}}=\mathcal{M}\left(e\left(p_{e}, \frac{\alpha}{2}\right)+\bar{e}\left(p_{\bar{e}},-\frac{\alpha}{2}\right) \rightarrow h\left(p_{h}\right)+t\left(p_{t}, \frac{\sigma}{2}\right)+\bar{t}\left(p_{\bar{t}}, \frac{\bar{\sigma}}{2}\right)\right),
$$

where $\alpha / 2= \pm 1 / 2$ gives the electron helicity, $\sigma / 2$ and $\bar{\sigma} / 2$ give the helicity of $t$ and $\bar{t}$ respectively in the $t \bar{t}$ rest frame. In order to fix our reference frame unambiguously, we start from the $e^{+} e^{-}$collision (laboratory) frame, where the four momenta are parameterized as

$$
\begin{aligned}
p_{e}^{\mu} & =\frac{\sqrt{s}}{2}(1,0,0,1) \\
p_{\bar{e}}^{\mu} & =\frac{\sqrt{s}}{2}(1,0,0,-1) \\
Q^{\mu} & =p_{t}^{\mu}+p_{\bar{t}}^{\mu}=\frac{\sqrt{s}}{2}\left(1+\frac{m_{t \bar{t}}^{2}-m_{h}^{2}}{s}, \bar{\beta} \sin \theta, 0, \bar{\beta} \cos \theta\right)=m_{t \bar{t}} \gamma(1, \beta \sin \theta, 0, \beta \cos \theta), \\
p_{h}^{\mu} & =\frac{\sqrt{s}}{2}\left(1+\frac{m_{h}^{2}-m_{t \bar{t}}^{2}}{s},-\bar{\beta} \sin \theta, 0,-\bar{\beta} \cos \theta\right)=\left(E_{h},-p_{h} \sin \theta, 0,-p_{h} \cos \theta\right)
\end{aligned}
$$

Here $m_{t \bar{t}}=\sqrt{Q^{2}}$ is the invariant mass of the $t \bar{t}$ system,

$$
\bar{\beta}=\frac{1}{s} \lambda^{1 / 2}\left(\sqrt{s}, m_{t \bar{t}}, m_{h}\right),
$$

with $\lambda(a, b, c)=(a+b+c)(a+b-c)(a-b+c)(a-b-c)$, and

$$
\gamma=\frac{E_{t \bar{t}}}{m_{t \bar{t}}}=\frac{\sqrt{s}}{2 m_{t \bar{t}}}\left(1+\frac{m_{t \bar{t}}^{2}-m_{h}^{2}}{s}\right), \quad \gamma \beta=\frac{p_{t \bar{t}}}{m_{t \bar{t}}}=\frac{\sqrt{s}}{2 m_{t \bar{t}}} \bar{\beta},
$$

are the Lorentz boost factors between the $e \bar{e}$ and the $t \bar{t}$ rest frames. The $t$ and $\bar{t}$ momenta are parameterized in the $t \bar{t}$ rest frame which is obtained from the laboratory frame by a rotation of $-\theta$ about the $y$-axis and then by a Lorentz boost along the $t \bar{t}$ momentum direction, which give

$$
\begin{aligned}
p_{t}^{\mu} & =\frac{m_{t \bar{t}}}{2}(1, \hat{\beta} \sin \hat{\theta} \cos \hat{\phi}, \hat{\beta} \sin \hat{\theta} \sin \hat{\phi}, \hat{\beta} \cos \hat{\theta}), \\
p_{\bar{t}}^{\mu} & =\frac{m_{t \bar{t}}}{2}(1,-\hat{\beta} \sin \hat{\theta} \cos \hat{\phi},-\hat{\beta} \sin \hat{\theta} \sin \hat{\phi},-\hat{\beta} \cos \hat{\theta}), \\
p_{h}^{\mu} & =\left(\sqrt{s} \gamma-m_{t \bar{t}}, 0,0,-\sqrt{s} \gamma \beta\right), \\
p_{e \bar{e}}^{\mu} & =(\sqrt{s} \gamma, 0,0,-\sqrt{s} \gamma \beta),
\end{aligned}
$$




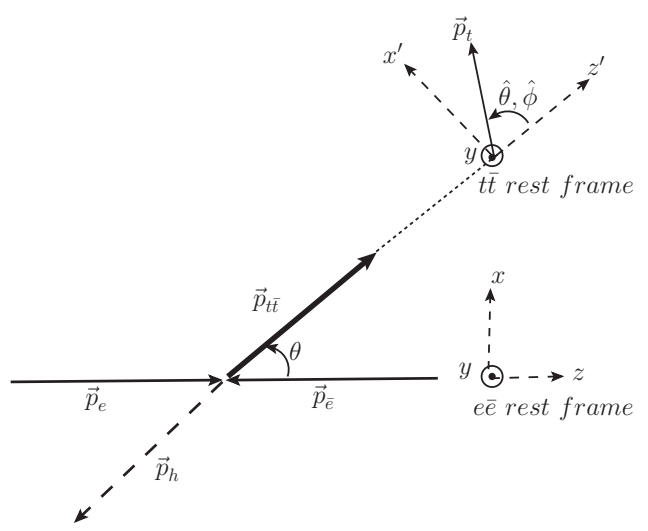

Figure 2. Kinematics of the $e \bar{e} \rightarrow h t \bar{t}$ process in the $e \bar{e}$ rest frame and in the $t \bar{t}$ rest frame.

with

$$
\hat{\beta}=\left(1-4 m_{t}^{2} / m_{t \bar{t}}^{2}\right)^{\frac{1}{2}}
$$

The $z$-axis is chosen along the negative of the common momentum direction of the Higgs boson and the $e^{+} e^{-}$system; see figure 2 . We find that the helicity amplitudes are most easily and systematically calculated in the frame obtained from the above by a rotation of $\hat{\phi}$ about $z$-axis, such that the top momentum has vanishing $y$-component:

$$
\begin{aligned}
p_{t}^{\mu} & =\frac{m_{t \bar{t}}}{2}(1, \hat{\beta} \sin \hat{\theta}, 0, \hat{\beta} \cos \hat{\theta}), \\
p_{\bar{t}}^{\mu} & =\frac{m_{t \bar{t}}}{2}(1,-\hat{\beta} \sin \hat{\theta}, 0,-\hat{\beta} \cos \hat{\theta}),
\end{aligned}
$$

the $h$ and $e^{+} e^{-}$four momenta are unchanged from eq. (2.5c) and (2.5d), whereas the initial $e$ and $\bar{e}$ momenta are now,

$$
\begin{aligned}
& p_{e}^{\mu}=\frac{\sqrt{s}}{2}(\gamma(1-\beta \cos \theta),-\sin \theta \cos \hat{\phi}, \sin \theta \sin \hat{\phi}, \gamma(\cos \theta-\beta)), \\
& p_{\bar{e}}^{\mu}=\frac{\sqrt{s}}{2}(\gamma(1+\beta \cos \theta), \sin \theta \cos \hat{\phi},-\sin \theta \sin \hat{\phi},-\gamma(\cos \theta+\beta)) .
\end{aligned}
$$

We calculate the helicity amplitudes in this specific $t \bar{t}$ rest frame where both the Higgs and the $e^{+} e^{-}$system have common momenta in the negative $z$-axis as in eq. (2.5c) and (2.5d), and the azimuthal angle $\hat{\phi}$ between the $t \bar{t}$ plane and the $e \bar{e}$ plane are given to the $e$ and $\bar{e}$ momenta.

We first note that the helicity amplitudes in eq. (2.1) can be factorized as

$$
\begin{aligned}
\mathcal{M}_{\alpha \sigma \bar{\sigma}} & =\sum_{V=\gamma, Z} D_{V}(q) L_{\mu}^{V}(e \bar{e} ; \alpha)\left(-g^{\mu \nu}\right) \hat{M}_{\nu}^{V}(h t \bar{t}, \sigma, \bar{\sigma}) \\
& =\sum_{V=\gamma, Z} D_{V}(q) L_{\mu}^{V}(e \bar{e} ; \alpha)\left(\sum_{\lambda} \epsilon^{\mu}(q, \lambda)^{*} \epsilon^{\nu}(q, \lambda)\right) \hat{M}_{\nu}^{V}(h t \bar{t}, \sigma, \bar{\sigma}) \\
& =\sum_{V=\gamma, Z} D_{V}(q) \sum_{\lambda} L_{\lambda \alpha}^{V}(e \bar{e}) \hat{M}_{\lambda \sigma \bar{\sigma}}^{V}(h t \bar{t})
\end{aligned}
$$


where $q^{\mu}=p_{e}^{\mu}+p_{\bar{e}}^{\mu}, \sqrt{s}$ is the center-of-mass energy, $D_{V}(q)=1 /\left(q^{2}-m_{V}^{2}+i m_{V} \Gamma_{V}\right)$ is the Breit-Wigner propagator factor for $\gamma, Z$, and also $W$ for later use. In eq. (2.9), the $\gamma$ and $Z$ polarization factor $-g^{\mu \nu}$ is replaced by the sum

$$
\sum_{\lambda} \epsilon^{\mu}(q, \lambda)^{*} \epsilon^{\nu}(q, \lambda)=-g^{\mu \nu}+\frac{q^{\mu} q^{\nu}}{q^{2}}
$$

with

$$
\epsilon^{\mu}(q, \lambda)= \begin{cases}\frac{1}{\sqrt{2}}(0, \pm 1,-i, 0) & \text { for } \lambda= \pm 1 \\ \gamma(\beta, 0,0,-1) & \text { for } \lambda=0\end{cases}
$$

along the $e \bar{e}$ four momentum $q^{\mu}=p_{e \bar{e}}^{\mu}=\sqrt{s} \gamma(1,0,0,-\beta)$, see eq. (2.5d), because of the $e \bar{e}$ current conservation,

$$
q^{\mu} L_{\mu}^{V}(e \bar{e} ; \alpha)=0
$$

in the $m_{e}=0$ limit. The leptonic currents are simply

$$
L_{\mu}^{V}(e \bar{e} ; \alpha)=g_{\alpha}^{V e e} v_{-\alpha}^{\dagger}(\bar{e}) \sigma_{\alpha}^{\mu} u_{\alpha}(e)
$$

where $u_{\alpha}$ and $v_{\alpha}$ are two-component chiral spinors with $\alpha=-1$ for $L(\alpha=+1$ for $R)$, and $\sigma_{ \pm}^{\mu}=(1, \pm \vec{\sigma})$ are the chiral four-vectors of $\sigma$ matrices. The gauge couplings are

$$
\begin{aligned}
& g_{-}^{Z f \bar{f}}=g_{z}\left(T_{f}^{3}-Q_{f} \sin ^{2} \theta_{W}\right), \quad g_{+}^{Z f \bar{f}}=-g_{z} Q_{f} \sin ^{2} \theta_{W} \\
& g_{-}^{\gamma f \bar{f}}=g_{+}^{\gamma f \bar{f}}=e Q_{f}=g_{V}^{\gamma f \bar{f}}
\end{aligned}
$$

with $g_{z}=g / \cos \theta_{W}$. We find

$$
L_{\mu}^{V}(e \bar{e} ; \alpha)=\alpha g_{\alpha}^{V e e} \sqrt{2 s} \epsilon_{\mu}(\vec{n}, \alpha)
$$

where the polarization direction $\vec{n}$ is along the $e$ three-momentum in the $e^{+} e^{-}$rest frame, which is obtained from eq. (2.10) by a Lorentz boost with $\beta$ and $\gamma$ given in eq. (2.4),

$$
\vec{n}=(-\sin \theta \cos \hat{\phi}, \sin \theta \sin \hat{\phi}, \cos \theta) .
$$

The leptonic amplitudes are hence expressed explicitly as

$$
L_{\lambda \alpha}^{V}(e \bar{e})=L_{\mu}^{V}(e \bar{e} ; \alpha) \epsilon^{\mu}(\vec{q}, \lambda)^{*}=\alpha g_{\alpha}^{V e e} \sqrt{2 s} \epsilon_{\mu}(\vec{n}, \alpha) \epsilon^{\mu}(\vec{q}, \lambda)^{*}
$$

in terms of Wigner's $D$ functions in the $e \bar{e}$ rest frame, where $\alpha$ denotes helicities along $\vec{n}$ in eq. (2.16), while $\lambda$ denotes those along $\vec{q}(-z)$ direction. Summing up we find

$$
\mathcal{M}_{\alpha \sigma \bar{\sigma}}=\sum_{V=\gamma, Z} R_{\alpha}^{V}\left[\frac{1-\alpha \cos \theta}{2} e^{-i \hat{\phi}} \hat{M}_{+\sigma \bar{\sigma}}^{V}+\frac{1+\alpha \cos \theta}{2} e^{i \hat{\phi}} \hat{M}_{-\sigma \bar{\sigma}}^{V}+\frac{\alpha \sin \theta}{\sqrt{2}} \hat{M}_{0 \sigma \bar{\sigma}}^{V}\right]
$$

with $R_{\alpha}^{V}=-\alpha g_{\alpha}^{V e e} \sqrt{2 s} D_{V}(q)$, where the three $D$ functions are shown explicitly. 
In this expression eq. (2.18), the leptonic amplitudes $L_{\lambda \alpha}^{V}(e \bar{e})$ gives the kinematical dependence on the production scattering angle $\theta$ and the azimuthal angle $\hat{\phi}$ in terms of Wigner's $D$ functions, whereas the $h t \bar{t}$ production amplitudes $\hat{M}_{\alpha \sigma \bar{\sigma}}^{V}$ depend only on the $t \bar{t}$ invariant mass $m_{t \bar{t}}$, and the polar angle of the top quark momentum $\hat{\theta}$. After using the equations of motion for $t$ and $\bar{t}$, we find a compact expression for $\hat{M}_{\alpha \sigma \bar{\sigma}}^{V}$,

$$
\begin{aligned}
\hat{\mathcal{M}}_{\lambda \sigma \bar{\sigma}}^{V}=\epsilon(q, \lambda)_{\nu} & \left\{2 m_{t} g_{h t t} \cos \xi_{h t t}\left(D_{t}^{1}+D_{t}^{2}\right)\left[g_{-}^{V t t} u_{L}^{\dagger} \sigma_{-}^{\nu} \nu_{L}+g_{+}^{V t t} u_{R}^{\dagger} \sigma_{+}^{\nu} v_{R}\right]\right. \\
+ & g_{h Z Z} \delta_{V Z} D_{Z}^{Q}\left[g_{-}^{Z t t} u_{L}^{\dagger} \sigma_{-}^{\nu} \nu_{L}+g_{+}^{Z t t} u_{R}^{\dagger} \sigma_{+}^{\nu} v_{R}+\frac{m Q^{\nu}}{m_{Z}^{2}} g_{A}^{Z t t}\left(u_{L}^{\dagger} v_{R}-u_{R}^{+} v_{L}\right)\right] \\
+ & g_{h t t} D_{t}^{1}\left[e^{-i \xi_{h t t}} g_{-}^{V t t} u_{R}^{\dagger} \sigma_{+} \cdot p_{h} \sigma_{-}^{\nu} v_{L}+e^{i \xi_{h t t}} g_{+}^{V t t} u_{L}^{\dagger} \sigma_{-} \cdot p_{h} \sigma_{+}^{\nu} v_{R}\right] \\
& \left.-g_{h t t} D_{t}^{2}\left[e^{i \xi_{h t t}} g_{-}^{V t t} u_{L}^{\dagger} \sigma_{-}^{\nu} \sigma_{+} \cdot p_{h} v_{R}+e^{-i \xi_{h t t}} g_{+}^{V t t} u_{R}^{\dagger} \sigma_{+}^{\nu} \sigma_{-} \cdot p_{h} v_{L}\right]\right\}
\end{aligned}
$$

where

$$
D_{t}^{1}=D_{t}\left(q-p_{t}\right), \quad D_{t}^{2}=D_{t}\left(p_{t}-q\right), \quad D_{Z}^{Q}=D_{Z}(Q) \quad \text { with } \quad Q=p_{t}+p_{\bar{t}}
$$

are the $t$ and $Z$ Breit-Wigner propagator factors.

For $\lambda= \pm 1$, the matrix elements are proportional to the Wigner's $D$ functions in the $t \bar{t}$ rest frame:

$$
\begin{aligned}
\hat{\mathcal{M}}_{\lambda \pm \mp}^{V}=\frac{ \pm 1-}{\sqrt{2} \cos \hat{\theta}}\left\{\left[4 m_{t} g_{h t t} \cos \xi_{h t t}\left(D_{t}^{1}+D_{t}^{2}\right)+2 g_{h Z Z} \delta_{V Z} D_{Z}^{Q}\right]\left(g_{V}^{V} \hat{E} \mp g_{A}^{V} \hat{p}\right)\right. \\
\left.+2 m_{t} g_{h t t} \cos \xi_{h t t}\left[g_{V}^{V}\left(D_{t}^{1}+D_{t}^{2}\right)\left(\sqrt{s} \gamma-m_{t \bar{t}}\right)-g_{A}^{V}\left(D_{t}^{1}-D_{t}^{2}\right) \lambda \sqrt{s} \gamma \beta\right)\right] \\
\left.+2 i m_{t} g_{h t t} \sin \xi_{h t t}\left[g_{V}^{V}\left(D_{t}^{1}+D_{t}^{2}\right) \lambda \sqrt{s} \gamma \beta-g_{A}^{V}\left(D_{t}^{1}-D_{t}^{2}\right)\left(\sqrt{s} \gamma-m_{t \bar{t}}\right)\right]\right\}
\end{aligned}
$$

for $(\sigma-\bar{\sigma}) / 2= \pm 1$, and

$$
\begin{aligned}
& \hat{M}_{\lambda \pm \pm}^{V}= \mp \lambda \frac{\sin \hat{\theta}}{\sqrt{2}}\left\{2 m_{t} g_{V}^{V}\left[2 m_{t} g_{h t t} \cos \xi_{h t t}\left(D_{t}^{1}+D_{t}^{2}\right)+g_{h Z Z} \delta_{V Z} D_{Z}^{Q}\right]\right. \\
&+2 g_{h t t} \cos \xi_{h t t}\left[g_{V}^{V}\left(D_{t}^{1}+D_{t}^{2}\right)\left(\hat{E}\left(\sqrt{s} \gamma-m_{t \bar{t}}\right) \mp \lambda \hat{p} \sqrt{s} \gamma \beta\right)\right. \\
&\left. \pm g_{A}^{V}\left(D_{t}^{1}-D_{t}^{2}\right)\left(\hat{p}\left(\sqrt{s} \gamma-m_{t \bar{t}}\right) \mp \lambda \hat{E} \sqrt{s} \gamma \beta\right)\right] \\
& \mp 2 i g_{h t t} \sin \xi_{h t t}\left[g_{V}^{V}\left(D_{t}^{1}+D_{t}^{2}\right)\left(\hat{p}\left(\sqrt{s} \gamma-m_{t \bar{t}}\right) \mp \lambda \hat{E} \sqrt{s} \gamma \beta\right)\right. \\
&\left.\left. \pm g_{A}^{V}\left(D_{t}^{1}-D_{t}^{2}\right)\left(\hat{E}\left(\sqrt{s} \gamma-m_{t \bar{t}}\right) \mp \lambda \hat{p} \sqrt{s} \gamma \beta\right)\right]\right\}
\end{aligned}
$$

for $(\sigma-\bar{\sigma}) / 2=0$. Here we introduce compact notation,

$$
g_{V}^{V}=\left(g_{-}^{V t t}+g_{+}^{V t t}\right) / 2, \quad g_{A}^{V}=\left(g_{-}^{V t t}-g_{+}^{V t t}\right) / 2,
$$


where

$$
\hat{E}=m_{t \bar{t}} / 2 \quad \text { and } \quad \hat{p}=\hat{E} \hat{\beta}
$$

are the top energy and momentum in the $t \bar{t}$ rest frame.

For $\lambda=0$, the amplitudes are still proportional to the Wigner's $D$ function when $(\sigma-\bar{\sigma}) / 2= \pm 1$, whereas they are the sum of the terms for the $J=1$ component and a constant term for the $J=0$ component when $(\sigma-\bar{\sigma}) / 2=0$ :

$$
\begin{aligned}
& \hat{\mathcal{M}}_{0 \pm \mp}^{V}=-\sin \hat{\theta}\left\{2 \gamma\left[2 m_{t} g_{h t t} \cos \xi_{h t t}\left(D_{t}^{1}+D_{t}^{2}\right)+g_{h Z Z} \delta_{V Z} D_{Z}^{Q}\right]\left(\hat{E} g_{V}^{V} \mp \hat{p} g_{A}^{V}\right)\right. \\
&\left.+2 g_{h t t} m_{t} \hat{E}_{h}\left[\cos \xi_{h t t} g_{V}^{V}\left(D_{t}^{1}+D_{t}^{2}\right)-i \sin \xi_{h t t} g_{A}^{V}\left(D_{t}^{1}-D_{t}^{2}\right)\right]\right\} \\
& \hat{\mathcal{M}}_{0 \pm \pm}^{V}= 4 m_{t}^{2} g_{h t t} \cos \xi_{h t t}\left(D_{t}^{1}+D_{t}^{2}\right) \gamma\left( \pm g_{V}^{V} \cos \hat{\theta}-g_{A}^{V} \beta\right) \\
&++2 m_{t} g_{h Z Z} \delta_{V Z} D_{Z}^{Q}\left[\gamma\left( \pm g_{V}^{V} \cos \hat{\theta}-g_{A}^{V} \beta\right)+\frac{m p_{h}}{m_{Z}^{2}} g_{A}^{V}\right] \\
&++2 g_{h t t} \cos \xi_{h t t}\left[\hat{E}\left( \pm E_{h} \cos \hat{\theta} g_{V}^{V}+p_{h} g_{A}^{V}\right)\left(D_{t}^{1}+D_{t}^{2}\right)\right. \\
&\left. \pm \hat{p}\left( \pm E_{h} \cos \hat{\theta} g_{A}^{V}+p_{h} g_{V}^{V}\right)\left(D_{t}^{1}-D_{t}^{2}\right)\right] \\
&-2 i g_{h t t} \sin \xi_{h t t}\left[ \pm \hat{p}\left( \pm E_{h} \cos \hat{\theta} g\right.\right. \\
&+ \\
&+\hat{E}\left( \pm E_{h} \cos \hat{\theta} g_{A}^{V}+p_{h}^{V} g_{V}^{V}\right)\left(D_{t}^{1}+D_{t}^{2}\right)
\end{aligned}
$$

Here, $E_{h}=\sqrt{s}-E_{t \bar{t}}=\sqrt{s}-m_{t \bar{t}} \gamma$ and $p_{h}=p_{t \bar{t}}=m_{t \bar{t}} \gamma \beta$ are the Higgs energy and the momentum in the $e \bar{e}$ rest frame. We note here that the terms proportional to $\sin \xi$ in all the 12 amplitudes eqs. (2.21), (2.22), (2.25), (2.26) are either proportional to $\hat{p}=\hat{E} \hat{\beta}, \beta$, or $D_{t}^{1}-D_{t}^{2}$, which are all suppressed strongly near the $h t \bar{t}$ production threshold.

It is instructive to note here that the above amplitudes satisfy the $\mathrm{CP}$ transformation properties

$$
M_{\alpha, \sigma, \bar{\sigma}}(\theta, \hat{\theta}, \hat{\phi} ; \xi)=M_{\alpha,-\bar{\sigma},-\sigma}(\pi-\theta, \pi-\hat{\theta},-\hat{\phi} ;-\xi)
$$

which is illustrated in figure 3. The upper plot (a) shows the three momenta and the helicities of $e$ and $\bar{e}$ in the $e \bar{e}$ rest frame, as well as those of $t$ and $\bar{t}$ in the $t \bar{t}$ rest frame. The common $z^{\prime}$-axis is chosen along the $t \bar{t}$ momentum direction in the $e \bar{e}$ rest frame. The electron momentum is in the $x^{\prime}-z^{\prime}$ plane. The vertical arrows $(\odot$ and $\otimes)$ show that the $t$-momentum has positive $y$-component, or $0<\hat{\theta}, \hat{\phi}<\pi / 2$. Shaded arrows show all the fermion spin directions for $\alpha=\sigma=\bar{\sigma}=+1$. The middle plot (b) is obtained from (a) by the $\mathrm{CP}$ transformation, which reverses the sign of all three momenta, but keeps the spin (hence the helicity is reserved), and exchange particles and antiparticles. Note that the initial $e \bar{e}$ state is invariant under CP transformation for each helicity. The momentum configuration of (b) is expressed in our reference frame as in the bottom plot (c), in which $\vec{p}_{t \bar{t}}$ is along the $z^{\prime}$ axis and the $y$ axis is along $\vec{p}_{e} \times \vec{p}_{t \bar{t}}$. The frame (c) is obtained from (b) 
(a)

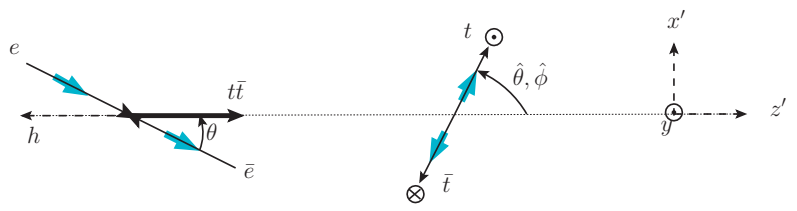

(b)

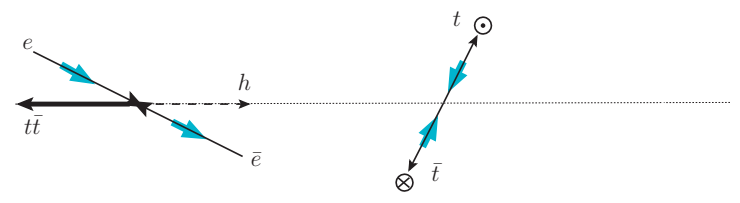

$(c)$

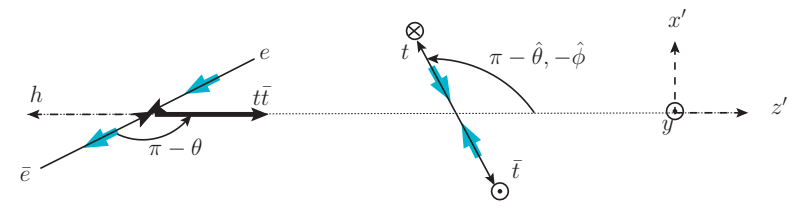

Figure 3. CP transformation of $e \bar{e} \rightarrow h t \bar{t}$ helicity amplitudes. Green shaded arrows show the spin direction along the particle momenta. The $e$ and $\bar{e}$ momenta are shown in the $e \bar{e}$ rest frame, whereas $t$ and $\bar{t}$ momenta are shown in the $t \bar{t}$ rest frame. The common $z^{\prime}$-axis is chosen along the $t \bar{t}$ momentum in the $e \bar{e}$ rest frame, or the negative of the $e \bar{e}$ and the $h$ momentum in the $t \bar{t}$ rest frame, and the common $y$-axis is vertical to the $e \bar{e} \rightarrow h+(t \bar{t})$ scattering plane; $\vec{n}_{y}=\frac{\vec{p}_{e} \times \vec{x}_{t \bar{t}}}{\left|\vec{p}_{e} \times \vec{p}_{t}\right|}$ in the $e \bar{e}$ rest frame. (a) shows the momenta and spins when the helicities of $e, t$ and $\bar{t}$ are all $+\frac{1}{2},(\alpha=\sigma=\bar{\sigma}=+)$. The symbol $\odot(\otimes)$ shows that the $y$-component of each momentum is positive (negative). Hence $0<\hat{\theta}, \hat{\phi}<\pi / 2$. (b) shows the process with $\mathrm{CP}$ transformed particles and their vectors in the original frames. (c) is obtained from (b) by two rotations, rotations of $\pi$ about the $z^{\prime}$-axis and by the $y$-axis. The coordinate system parameterizing the three momentum is the same as in (a), and we can read off the CP transformation of the helicity amplitudes as given by eq. (2.27).

by rotations, which do not affect the helicity amplitudes. This gives the identity eq. (2.27), which show explicitly how CP-violating term proportional to $\sin \xi_{h t t}$ should behave under exchange of angular variables:

$$
(\theta, \hat{\theta}, \hat{\phi}) \leftrightarrow(\pi-\theta, \pi-\hat{\theta},-\hat{\phi}),
$$

and when the $t$ and $\bar{t}$ helicities are exchanged as

$$
(\sigma, \bar{\sigma}) \leftrightarrow(-\bar{\sigma},-\sigma)
$$

The helicity amplitudes $\hat{M}_{\alpha \sigma \bar{\sigma}}^{V}$ contain all the information about the $h t \bar{t}$ (and the $h Z Z$ ) coupling that we can probe in the process $e^{+} e^{-} \rightarrow h t \bar{t}$. Being complex numbers, however, they are not directly observable in experiments. For instance, the differential cross section

$$
d \sigma=\frac{1}{2 s} \frac{1}{4} \sum_{\alpha} \sum_{\sigma} \sum_{\bar{\sigma}}\left|M_{\alpha \sigma \bar{\sigma}}\right|^{2} d \Phi_{h t t}
$$

with the 3-body phase space

$$
d \Phi_{h t t}=\frac{1}{64 \pi^{2}} \bar{\beta} \hat{\beta} \frac{d m_{t \bar{t}}^{2}}{2 \pi} \frac{d \cos \theta d \cos \hat{\theta} d \hat{\phi}}{8 \pi}
$$


with $\bar{\beta}$ in eq. (2.3) and $\hat{\beta}$ in eq. (2.6), measures only the squared sum of all the helicity amplitudes. With $e^{-}$(and possibly $e^{+}$) beam polarization, the sum of $e_{L} \bar{e}_{R}$ annihilation $(\alpha=-1)$ and that of $e_{R} \bar{e}_{L}$ annihilation $(\alpha=+1)$ can be resolved.

When we study $t$ and $\bar{t}$ decay distributions and their correlations, we can measure 15 more combinations of the helicity amplitudes (16 each, including the absolute value squared of the 4 amplitudes for $\alpha=-1$ and $\alpha=+1$ with beam polarization). In this section, we illustrate this when both $t$ and $\bar{t}$ decay semi-leptonically,

$$
e^{+} e^{-} \rightarrow h t \bar{t} \rightarrow h\left(b \ell \bar{\nu}_{\ell}\right)\left(\bar{b} \ell^{\prime} \bar{\nu}_{\ell^{\prime}}\right)
$$

The helicity amplitude for the process can be expressed (for $e_{\alpha} \bar{e}_{-\alpha}$ collisions) as

$$
\mathcal{M}_{\alpha}=\sum_{\sigma} \sum_{\bar{\sigma}} M_{\alpha \sigma \bar{\sigma}} D_{t}\left(p_{t}\right) D_{t}\left(p_{\bar{t}}\right) M_{\sigma} \bar{M}_{\bar{\sigma}}
$$

with the Breit-Wigner propagator factors

$$
D_{t}(p)=\frac{1}{p^{2}-m_{t}^{2}+i m_{t} \Gamma_{t}}
$$

and the decay amplitudes

$$
\begin{aligned}
& M_{\sigma}=\frac{g^{2}}{2} D_{W}\left(p_{t}-p_{b}\right) u_{L}^{\dagger}\left(p_{b}\right) \sigma_{-}^{\mu} u_{L}\left(p_{t}, \sigma\right) u_{L}^{\dagger}\left(p_{\nu}\right) \sigma_{-\mu} v_{L}\left(p_{\bar{\ell}}\right) \\
& \bar{M}_{\bar{\sigma}}=\frac{g^{2}}{2} D_{W}\left(p_{\bar{t}}-p_{\bar{b}}\right) v_{L}^{\dagger}\left(p_{\bar{t}}, \bar{\sigma}\right) \sigma_{-}^{\mu} v_{L}\left(p_{\bar{b}}\right) u_{L}^{\dagger}\left(p_{\ell}\right) \sigma_{-\mu} v_{L}\left(p_{\bar{\nu}}\right)
\end{aligned}
$$

The differential cross section for the process eq. (2.32) is hence (for unpolarized beams)

$$
d \sigma=\frac{1}{2 s} \frac{1}{4} \sum_{\alpha}\left|\mathcal{M}_{\alpha}\right|^{2} d \Phi_{7}
$$

where the 7-body phase space can be decomposed as

$$
d \Phi_{7}=d \Phi_{h t t}\left(m_{h}^{2}, p_{t}^{2}, p_{\bar{t}}^{2}\right) \frac{d p_{t}^{2}}{2 \pi} \frac{d p_{\bar{t}}^{2}}{2 \pi} d \Phi_{b \bar{\ell}_{\ell}}\left(p_{t}^{2}\right) d \Phi_{\bar{b}^{\prime} \bar{\nu}_{\ell^{\prime}}}\left(p_{\bar{t}}^{2}\right)
$$

with $p_{t}^{2}$ and $p_{\bar{t}}^{2}$ as the invariant mass squared of the $\left(b \bar{\ell} \nu_{\bar{\ell}}\right)$ and $\left(\bar{b} \ell^{\prime} \bar{\nu}_{\bar{\ell}^{\prime}}\right)$ systems, respectively. In the narrow width limit of the top quark,

$$
\int \frac{d p_{t}^{2}}{2 \pi}\left|D_{t}\left(p_{t}\right)\right|^{2}=\int \frac{d p_{\bar{t}}^{2}}{2 \pi}\left|D_{t}\left(p_{\bar{t}}\right)\right|^{2}=\frac{1}{2 m_{t} \Gamma_{t}}
$$

holds and the differential cross section in eq. (2.36) can be expressed as

$$
d \sigma=\frac{1}{2 s} \frac{1}{4} \sum_{\alpha}\left|\sum_{\sigma} \sum_{\bar{\sigma}} M_{\alpha \sigma \bar{\sigma}} M_{\sigma} \bar{M}_{\bar{\sigma}}\right|^{2} d \Phi_{h t \bar{t}} \frac{d \Phi_{b \bar{\ell}_{\ell}}}{2 m_{t} \Gamma_{t}} \frac{d \Phi_{\bar{b}^{\prime} \bar{\nu}_{\ell^{\prime}}}}{2 m_{t} \Gamma_{t}} .
$$

The above expression can be expressed as

$$
d \sigma=\sum_{\sigma} \sum_{\bar{\sigma}} \sum_{\sigma^{\prime}} \sum_{\bar{\sigma}^{\prime}}\left(\frac{1}{2 s} \frac{1}{4} \sum_{\alpha}\left(M_{\alpha \sigma \bar{\sigma}}\right)\left(M_{\alpha \sigma^{\prime} \bar{\sigma}^{\prime}}\right)^{*} d \Phi_{h t \bar{t}}\right) d \rho_{\sigma \sigma^{\prime}} d \bar{\rho}_{\bar{\sigma} \bar{\sigma}^{\prime}}
$$


with

$$
\begin{aligned}
& d \rho_{\sigma \sigma^{\prime}}=M_{\sigma} M_{\sigma^{\prime}}^{*} d \Phi_{b \bar{\nu}_{\ell}} /\left(2 m_{t} \Gamma_{t}\right), \\
& d \bar{\rho}_{\bar{\sigma} \bar{\sigma}^{\prime}}=M_{\bar{\sigma}} M_{\bar{\sigma}^{\prime}}^{*} d \Phi_{\bar{b} \ell^{\prime} \bar{\nu}_{\ell^{\prime}}} /\left(2 m_{t} \Gamma_{t}\right) .
\end{aligned}
$$

The differential decay density matrices in eqs. (2.41) are calculated in appendix A, and take particularly simple form for semi-leptonic decay

$$
\begin{aligned}
& d \rho=\left(\begin{array}{l}
d \rho_{++} d \rho_{+-} \\
d \rho_{-+} d \rho_{--}
\end{array}\right)=\left(\begin{array}{cc}
1+\cos \bar{\theta}^{*} & \sin \bar{\theta}^{*} e^{i \bar{\phi}^{*}} \\
\sin \bar{\theta}^{*} e^{-i \bar{\phi}^{*}} & 1-\cos \bar{\theta}^{*}
\end{array}\right) B_{\ell} \frac{d \cos \bar{\theta}^{*} d \bar{\phi}^{*}}{4 \pi} \\
& d \bar{\rho}=\left(\begin{array}{l}
d \bar{\rho}_{++} d \bar{\rho}_{+-} \\
d \bar{\rho}_{-+} d \bar{\rho}_{--}
\end{array}\right)=\left(\begin{array}{cc}
1+\cos \theta^{*} & \sin \theta^{*} e^{-i \phi^{*}} \\
\sin \theta^{*} e^{i \phi^{*}} & 1-\cos \theta^{*}
\end{array}\right) B_{\ell} \frac{d \cos \theta^{*} d \phi^{*}}{4 \pi}
\end{aligned}
$$

where $B_{\ell}=\sum_{\ell} B\left(t \rightarrow b \bar{\ell} \nu_{\ell}\right)=\sum_{\ell} B\left(\bar{t} \rightarrow \bar{b} \ell \bar{\nu}_{\ell}\right) \simeq 0.33$ is the semi-leptonic branching fractions summed over $\ell=e, \mu, \tau$. Here, $\bar{\theta}^{*}$ and $\bar{\phi}^{*}\left(\theta^{*}\right.$ and $\left.\phi^{*}\right)$ are the polar and azimuthal angles of $\bar{\ell}(\ell)$ in the $t(\bar{t})$ rest frame where the polar axis is chosen along the $t$ momentum direction in the $t \bar{t}$ rest frame. More details are explained in appendix A. By inserting eq. (2.42) into eq. (2.40), we find

$$
\begin{aligned}
& \frac{d \sigma_{\alpha}}{d \Phi_{h t t} d \cos \bar{\theta}^{*} d \bar{\phi}^{*} d \cos \theta^{*} d \phi^{*}} \\
& =\frac{B_{\ell}^{2}}{(4 \pi)^{2}} \times\left\{\left|M_{\alpha++}\right|^{2}\left(1+\cos \bar{\theta}^{*}\right)\left(1+\cos \theta^{*}\right)+\left|M_{\alpha+-}\right|^{2}\left(1+\cos \bar{\theta}^{*}\right)\left(1-\cos \theta^{*}\right)\right. \\
& \quad+\left|M_{\alpha--}\right|^{2}\left(1-\cos \bar{\theta}^{*}\right)\left(1-\cos \theta^{*}\right)+\left|M_{\alpha-+}\right|^{2}\left(1-\cos \bar{\theta}^{*}\right)\left(1+\cos \theta^{*}\right) \\
& \quad+2\left[\operatorname{Re}\left(M_{\alpha++} M_{\alpha+-}^{*}\right) \cos \phi^{*}+\operatorname{Im}\left(M_{\alpha++} M_{\alpha+-}^{*}\right) \sin \phi^{*}\right] \sin \theta^{*}\left(1+\cos \bar{\theta}^{*}\right) \\
& \quad+2\left[\operatorname{Re}\left(M_{\alpha-+} M_{\alpha--}^{*}\right) \cos \phi^{*}+\operatorname{Im}\left(M_{\alpha-+} M_{\alpha--}^{*}\right) \sin \phi^{*}\right] \sin \theta^{*}\left(1-\cos \bar{\theta}^{*}\right) \\
& \quad+2\left[\operatorname{Re}\left(M_{\alpha++} M_{\alpha-+}^{*}\right) \cos \bar{\phi}^{*}-\operatorname{Im}\left(M_{\alpha++} M_{\alpha-+}^{*}\right) \sin \bar{\phi}^{*}\right] \sin \bar{\theta}^{*}\left(1+\cos \theta^{*}\right) \\
& \quad+2\left[\operatorname{Re}\left(M_{\alpha+-} M_{\alpha--}^{*}\right) \cos \bar{\phi}^{*}-\operatorname{Im}\left(M_{\alpha+-} M_{\alpha--}^{*}\right) \sin \bar{\phi}^{*}\right] \sin \bar{\theta}^{*}\left(1-\cos \theta^{*}\right) \\
& \quad+2\left[\operatorname{Re}\left(M_{\alpha++} M_{\alpha--}^{*}\right) \cos \left(\bar{\phi}^{*}-\phi^{*}\right)-\operatorname{Im}\left(M_{\alpha++} M_{\alpha--}^{*}\right) \sin \left(\bar{\phi}^{*}-\phi^{*}\right)\right] \sin \bar{\theta}^{*} \sin \theta^{*} \\
& \left.\quad+2\left[\operatorname{Re}\left(M_{\alpha+-} M_{\alpha-+}^{*}\right) \cos \left(\bar{\phi}^{*}+\phi^{*}\right)-\operatorname{Im}\left(M_{\alpha+-} M_{\alpha-+}^{*}\right) \sin \left(\bar{\phi}^{*}+\phi^{*}\right)\right] \sin \bar{\theta}^{*} \sin \theta^{*}\right\} .
\end{aligned}
$$

It is now clear that all the real and imaginary parts of the product of the helicity amplitudes $M_{\alpha \sigma \bar{\sigma}}$ and its complex conjugates $M_{\alpha \sigma^{\prime} \bar{\sigma}^{\prime}}^{*}$ including $\sigma^{\prime} \neq \sigma$ and $\bar{\sigma}^{\prime} \neq \bar{\sigma}$ can be measured by studying the correlated decays $t \rightarrow b \bar{\ell} \nu$ and $\bar{t} \rightarrow \bar{b} \ell \bar{\nu}$ at all $h t \bar{t}$ phase space point $\left(m_{t \bar{t}}, \cos \theta, \cos \hat{\theta}, \hat{\phi}\right)$. There are four $\sum_{\alpha}\left|M_{\alpha \sigma \bar{\sigma}}\right|^{2}$ terms, six $\sum_{\alpha} \operatorname{Re}\left(M_{\alpha \sigma^{\prime} \bar{\sigma}^{\prime}} M_{\alpha \sigma^{\prime} \bar{\sigma}^{\prime}}^{*}\right)$ terms and $\operatorname{six} \sum_{\alpha} \operatorname{Im}\left(M_{\alpha \sigma \bar{\sigma}} M_{\alpha \sigma^{\prime} \bar{\sigma}^{\prime}}^{*}\right)$ terms. With polarized $e$ beams, $\alpha=-1$ and $\alpha=+1$ combinations can be resolved.

In figure 4, we show the $\hat{\phi}$ distribution of all the 16 combinations of $M_{\alpha \sigma \bar{\sigma}} M_{\alpha \sigma^{\prime} \bar{\sigma}^{\prime}}^{*}$ for $\alpha=-1$ case $\left(e_{L} \bar{e}_{R}\right.$ annihilation) at $\sqrt{s}=500 \mathrm{GeV}$ (left) and at $\sqrt{s}=1000 \mathrm{GeV}$ (right). We set $\cos \theta=0, \cos \hat{\theta}=0.5$ and $m_{t \bar{t}}=350 \mathrm{GeV}$ at both energies where the $\xi_{h t t}$-dependences are found to be significant. We compare $\xi_{h t t}=\frac{\pi}{4}$ (solid lines) and $\xi_{h t t}=-\frac{\pi}{4}$ (dashed lines) for $\sqrt{s}=500 \mathrm{GeV}$, and $\xi_{h t t}= \pm 0.2$ for $\sqrt{s}=1000 \mathrm{GeV}$. 

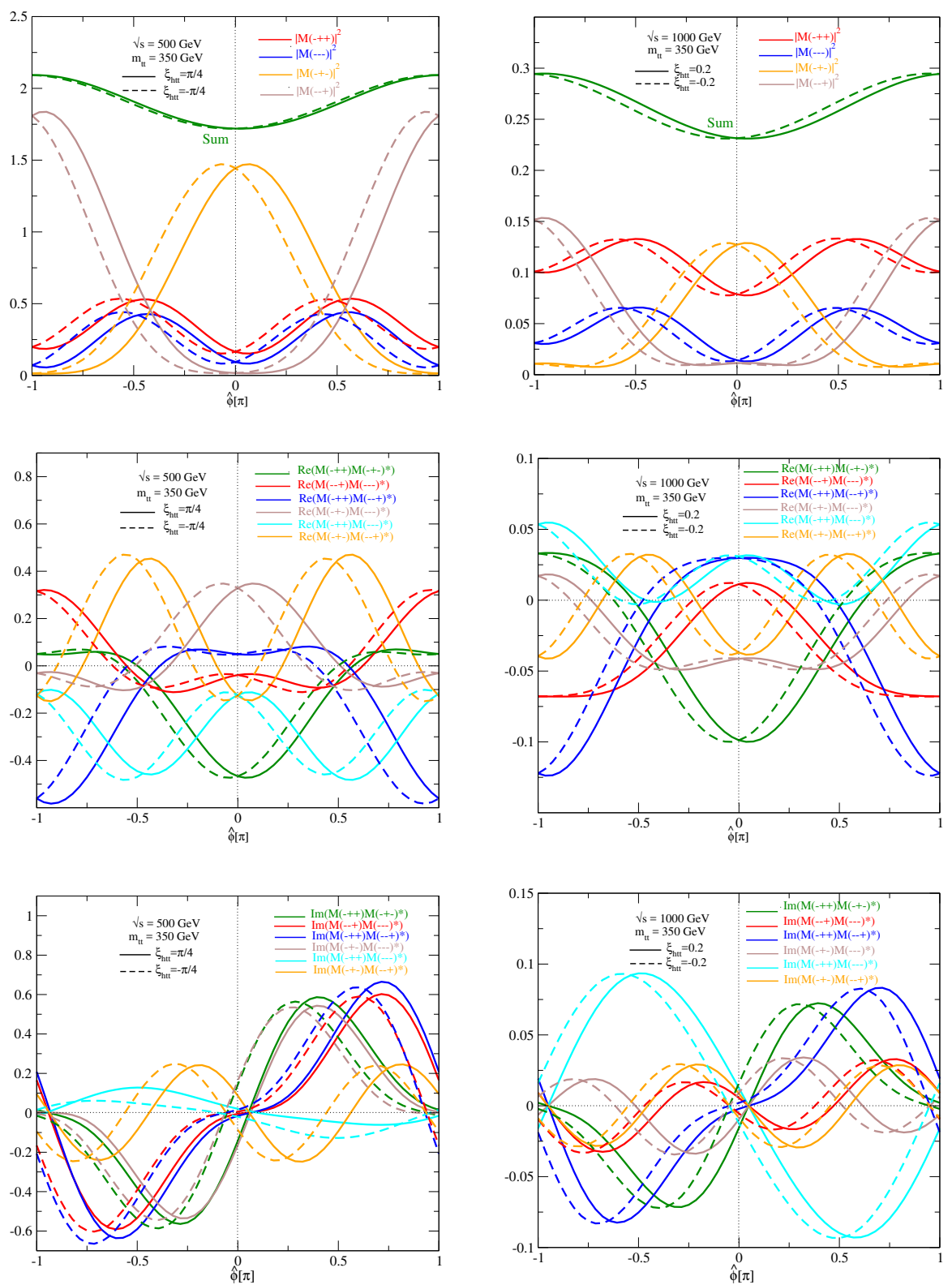

Figure 4. The azimuthal angle distribution of the 16 helicity amplitudes combinations $M_{\alpha \sigma \bar{\sigma}} M_{\alpha \sigma^{\prime} \bar{\sigma}^{\prime}}^{*}$ for $\alpha=-1$ ( $e_{L} \bar{e}_{R}$ annihilation) at $\sqrt{s}=500 \mathrm{GeV}$ for $\xi_{h t t}= \pm \pi / 4$ (left panels) and at $\sqrt{s}=$ $1000 \mathrm{GeV}$ for $\xi_{h t t}= \pm 0.2$ (right panels). We set $\cos \theta=0, \cos \hat{\theta}=0.5$ and $m_{t \bar{t}}=350 \mathrm{GeV}$ in all the plots. Solid curves are for $\xi_{h t t}>0$, and dashed curves are for $\xi_{h t t}<0$.

The top panels show the four absolute value squared $\left|M_{-\sigma \bar{\sigma}}\right|^{2}$ and their sum. CP violation appears as a phase shift in the $\hat{\phi}$ distribution whose sign and magnitude are proportional to $\xi_{h t t}[16]$. The difference is reduced significantly when only the total sum of all squared amplitudes, i.e. the $h t \bar{t}$ distributions are observed. The polar angle distributions of $t$ and $\bar{t}$ decays can resolve the four individual contributions $(\sigma \bar{\sigma})=$ $(+,-),(-,+),(++),(--)$, according to eq. (2.43). 
The middle panels show the real part of the six off-diagonal $\left(\sigma^{\prime} \neq \sigma\right.$ or $\left.\bar{\sigma}^{\prime} \neq \bar{\sigma}\right)$ products. In the absence of CP violation, these are even functions of $\hat{\phi}$. The CP-violating asymmetries appear again as a phase shift or asymmetries between $\hat{\phi}>0$ and $\hat{\phi}<0$. From eq. (2.43), we learn that the 6 real terms are measured as coefficients of $\cos \phi^{*}, \cos \bar{\phi}^{*}$, $\cos \left(\bar{\phi}^{*}+\phi^{*}\right)$ or $\cos \left(\bar{\phi}^{*}-\phi^{*}\right)$.

In the bottom panel we show the corresponding imaginary parts of the six off-diagonal products. They are measured as coefficients of $\sin \phi^{*}, \sin \bar{\phi}^{*}, \sin \left(\bar{\phi}^{*}+\phi^{*}\right)$ or $\sin \left(\bar{\phi}^{*}-\phi^{*}\right)$ according to eq. (2.43). It is worth noting that these distributions are odd functions of $\hat{\phi}$ if there is no $\mathrm{CP}$ violation $\left(\xi_{h t t}=0\right)$. CP violation induces a phase shift in these distributions whose sign and magnitude are proportional to $\xi_{h t t}$.

We also study all the distributions for $\alpha=+1\left(e_{R} \bar{e}_{L}\right.$ annihilation), but they are found to be very similar to the $\alpha=-1$ case shown in figure 4 , with significantly smaller magnitudes. Although the results shown in figure 4 are for a particular kinematical configuration of $h t \bar{t}\left(m_{t \bar{t}}=350 \mathrm{GeV}, \cos \theta=0, \cos \hat{\theta}=0.5\right)$, and for $\alpha=-1\left(e_{L} \bar{e}_{R}\right.$ annihilation), their dependence on the sign of $\xi_{h t t}$ shows the possible improvement in the CP-violation discovery potential in $e^{+} e^{-}$collision experiments, by making use of all the information given by $t$ and $\bar{t}$ decay angular correlations.

\section{Cross sections and QCD corrections}

In this section we study the energy dependence of the total cross sections and the QCD higher-order corrections, perturbative NLO corrections and resummation of Coulombic corrections that account for topponium formation below and around the threshold, $m_{t \bar{t}} \sim$ $2 m_{t}$. Those studies are made in the two CP-conserving limits, at $\xi_{h t t}=0$ ( $h=H$, the SM limit), and at $\xi_{h t t}=\frac{\pi}{2}$ ( $h=A$, the pseudo scalar limit). The results are used to normalize our statistical analysis in the next sections, which are based on the leading-order (LO) matrix elements.

\subsection{Leading-order production cross section}

We show in figure 5 the leading-order total cross section of the $e^{+} e^{-} \rightarrow h t \bar{t}$ process in the two limits, the pure CP-even $(h=H)$ limit when $\xi_{h t t}=0, \kappa_{h t t}=\kappa_{h Z Z}=1$, and the pure CP-odd $(h=A)$ limit when $\xi_{h t t}=\frac{\pi}{2}, \kappa_{h t t}=1, \kappa_{h Z Z}=0 . \sigma(H t \bar{t})$ is about $0.28 \mathrm{fb}$ at $\sqrt{s}=500 \mathrm{GeV}$, reaching $2 \mathrm{fb}$ at $\sqrt{s} \sim 600 \mathrm{GeV}$ and stays above $2 \mathrm{fb}$ until $\sqrt{s} \sim 1 \mathrm{TeV}$. On the other hand, $\sigma(A t \bar{t})$ is about $0.0045 \mathrm{fb}$ (below the scale of figure 5 ) at $\sqrt{s}=500 \mathrm{GeV}$, rising quickly with energy, reaching $0.43 \mathrm{fb}$ at $\sqrt{s}=1000 \mathrm{GeV}$.

Because the CP-violating asymmetries appear as interference effects between CPeven and CP-odd amplitudes, we show also the ratio of the two cross sections, $R=$ $\sigma(A t \bar{t}) / \sigma(H t \bar{t})$ in figure 5. We can very roughly expect that the CP asymmetry is proportional to $\sqrt{R}$. The scale of $R$ is given along the right-hand vertical axis. It grows rapidly from 0.016 at $\sqrt{s}=500 \mathrm{GeV}$ to 0.047 at $\sqrt{s}=550 \mathrm{GeV}$, reaching 0.2 at $\sqrt{s}=1000 \mathrm{GeV}$.

\subsection{QCD corrections}

Shown in figure 6 are the differential cross sections $d \sigma / d m_{t \bar{t}}$ v.s. $m_{t \bar{t}}$ for $h=H$ (black solid curves) and for $h=A$ (red dashed curves) at $\sqrt{s}=500 \mathrm{GeV}$ (left) and at $\sqrt{s}=1000 \mathrm{GeV}$ 


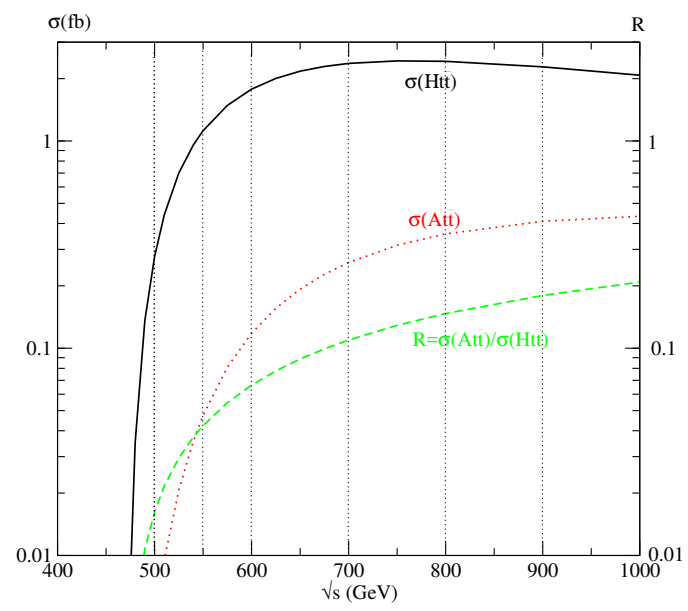

Figure 5. The leading-order total cross section for $e^{+} e^{-} \rightarrow h t \bar{t}$ for the pure CP-even $(h=H)$ and CP-odd $(h=A)$ limits. $\sigma(H t \bar{t})$ and $\sigma(A t \bar{t})$ are shown by black solid and red dotted curves respectively, and their ratio $R=\sigma(A t \bar{t}) / \sigma(H t \bar{t})$ is given by green dashed line.
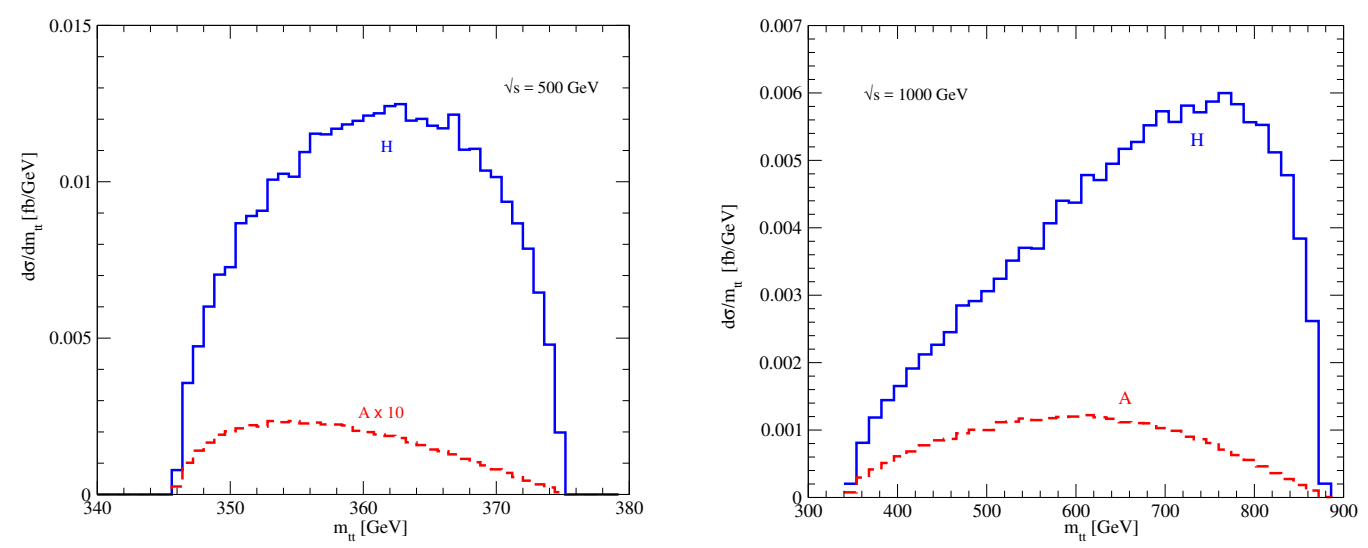

Figure 6. $m_{t \bar{t}}$ distributions for $H$ and $A$ at $\sqrt{s}=500 \mathrm{GeV}$ (left) and $1000 \mathrm{GeV}$ (right) in the leading order.

(right) calculated in the leading order. The $h=A$ cross section at $\sqrt{s}=500 \mathrm{GeV}$ is multiplied by 10, in order to show the shape difference between the CP-even $(h=H)$ and CP-odd $(h=A)$ limits. It is clear from the two cases shown in the figure that the ratio of the CP-odd and CP-even amplitudes squared is large at low $m_{t \bar{t}}$, at all energies. This suggests that the sensitivity to $\mathrm{CP}$ asymmetry is high at low $m_{t \bar{t}}$, and hence the corrections including topponium formation can have significant impacts on our study of $\mathrm{CP}$ violation in the top Yukawa coupling.

We show in figure 7 the differential cross section $d \sigma / d m_{t \bar{t}}$ for the SM Higgs boson $(h=H)$ with QCD corrections. NLO QCD corrections to the process are evaluated by using MadGraph5_aMC@NLO [18]. In addition, we also consider the corrections by Coulomb resummation [16, 19-21]. According to refs. [21, 22], we estimate the Coulomb resummation corrections as follows. First, we evaluate the Born-level helicity amplitudes by including the decays of top-quarks, $e^{+} e^{-} \rightarrow h t \bar{t} \rightarrow h b W^{+} \bar{b} W^{-}$, in which the top-quarks can 

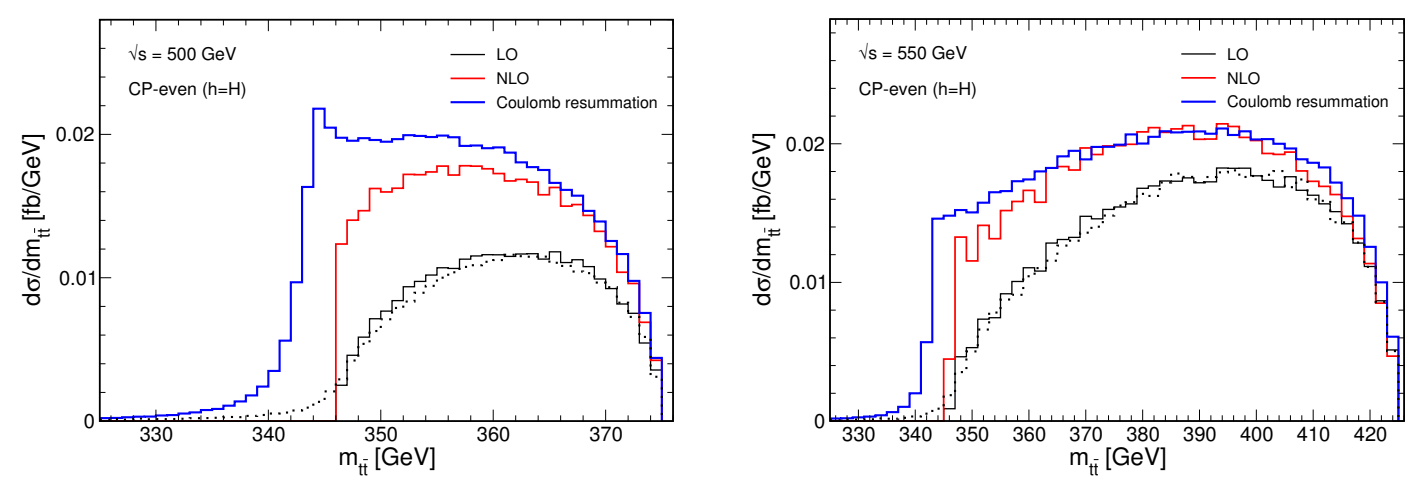

Figure 7. $m_{t \bar{t}}$ distribution for the pure scalar case $(h=H)$ at $\sqrt{s}=500 \mathrm{GeV}$ and $550 \mathrm{GeV}$. Black solid and red solid lines are the LO and NLO cross sections for the on-shell $t \bar{t}$, respectively, and black dotted and blue solid lines are the Born-level and the Coulomb-resummed cross sections with top-quark off-shellness, respectively.
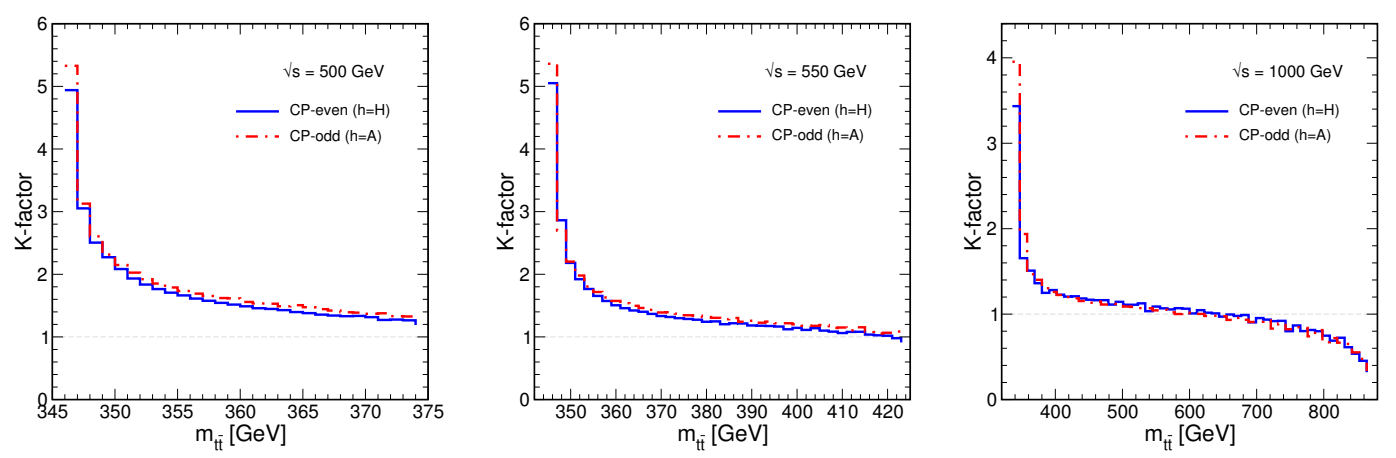

Figure 8. $m_{t \bar{t}}$ dependence of the NLO QCD $K$-factors in the $e^{+} e^{-} \rightarrow h t \bar{t}$ process for the CP-even $(h=H)$ and CP-odd $(h=A)$ limits at $\sqrt{s}=500 \mathrm{GeV}, 550 \mathrm{GeV}$, and $1000 \mathrm{GeV}$.

be off-shell and $m_{t \bar{t}}$ can be below $2 m_{t}$. Then, the amplitudes are corrected by multiplying with the $S$-wave Green function for non-relativistic $t \bar{t}$ with the energy $E=m_{t \bar{t}}-2 m_{t}$. This prescription is justified by the fact that near the $t \bar{t}$ threshold where the Coulomb resummation is important the amplitudes are dominated by the $S$-wave component. To evaluate the Green function we employ the NLO QCD potential with the renormalization scale $\mu=40 \mathrm{GeV}$. Finally, the squared amplitudes are corrected by a hard correction factor $K \simeq 0.7$ which is numerically extracted by matching with the NLO cross section at an intermediate $m_{t \bar{t}}$.

In figure 7 , we show the differential cross section for the pure scalar case $(h=H)$ at $\sqrt{s}=500 \mathrm{GeV}$ (left) and $550 \mathrm{GeV}$ (right). LO and NLO cross sections for the on-shell $t \bar{t}$ limit are plotted by black solid and red solid lines, respectively. Born-level and the Coulomb-resummed cross sections with top-quark off-shellness are plotted by black dotted and blue solid lines, respectively. At $\sqrt{s}=500 \mathrm{GeV}$, the NLO corrections enhance the total cross section by around $60 \%$, while the Coulomb resummation (including the NLO effects) enhances the cross section by about $120 \%$. At $\sqrt{s}=550 \mathrm{GeV}$, the total cross section is enhanced by $20 \%$ and $40 \%$, by the NLO and Coulomb resummation corrections, respectively. 


\begin{tabular}{|r|cc|cc|}
\hline$\sqrt{s}$ & \multicolumn{2}{|c|}{ CP-even $(h=H)$} & \multicolumn{2}{c|}{ CP-odd $(h=A)$} \\
{$[\mathrm{GeV}]$} & NLO & Coulomb resum & NLO & Coulomb resum \\
\hline 500 & 1.57 & 2.18 & 1.76 & 2.89 \\
550 & 1.26 & 1.39 & 1.36 & 1.60 \\
1000 & 0.95 & - & 1.05 & - \\
\hline
\end{tabular}

Table 1. $K$-factors by NLO and Coulomb-resummation corrections to the total cross sections of the process $e^{+} e^{-} \rightarrow h t \bar{t}$ for the CP-even $(h=H)$ and CP-odd $(h=A)$ cases, at $\sqrt{s}=500 \mathrm{GeV}$, $550 \mathrm{GeV}$, and $1000 \mathrm{GeV}$.

\begin{tabular}{|r|ccc|ccc|}
\hline \multicolumn{1}{|c|}{$\sqrt{s}$} & \multicolumn{2}{|c|}{$\sigma(H t \bar{t})[\mathrm{fb}]$} & \multicolumn{3}{c|}{$\sigma(A t \bar{t})[\mathrm{fb}]$} \\
{$[\mathrm{GeV}]$} & LO & NLO & with Coulomb resum & LO & NLO & with Coulomb resum \\
\hline 500 & 0.28 & 0.44 & 0.61 & 0.0045 & 0.0079 & 0.0130 \\
550 & 1.12 & 1.41 & 1.56 & 0.047 & 0.064 & 0.075 \\
1000 & 2.08 & 1.97 & - & 0.43 & 0.45 & - \\
\hline
\end{tabular}

Table 2. $e^{+} e^{-} \rightarrow h t \bar{t}$ cross sections at LO, NLO and with Coulomb-resummation corrections for the pure CP-even $(h=H)$ and CP-odd $(h=A)$ limits at $\sqrt{s}=500 \mathrm{GeV}, 550 \mathrm{GeV}$, and $1000 \mathrm{GeV}$.

In figure 8 , we plot the ratio of the NLO cross section to the LO cross section as a function of $m_{t \bar{t}}$ for the pure scalar $(h=H)$ and pseudoscalar $(h=A)$ cases at $\sqrt{s}=$ $500 \mathrm{GeV}, 550 \mathrm{GeV}$, and $1000 \mathrm{GeV}$. We find the $K$-factors for the $m_{t \bar{t}}$ distribution are almost the same for the scalar and pseudoscalar processes. QCD corrections are large near the threshold, $m_{t \bar{t}} \simeq 2 m_{t}$, because of the Coulomb singularity, but become almost flat and small at large $m_{t \bar{t}}$.

In table 1 , we list the $K$-factors of the total $h t \bar{t}$ production cross section by QCD correction for the pure scalar $(h=H)$ and pseudoscalar $(h=A)$ limits at $\sqrt{s}=500 \mathrm{GeV}$, $550 \mathrm{GeV}$, and $1000 \mathrm{GeV}$. An evaluation of the Coulomb resummation at $\sqrt{s}=1000 \mathrm{GeV}$ is omitted because it has negligible effects. Both the NLO corrections and Coulomb resummation (which contain NLO) corrections are largest at $\sqrt{s}=500 \mathrm{GeV}$, still significant at $550 \mathrm{GeV}$, but small at $1000 \mathrm{GeV}$. The enhancement factors are larger for CP-odd case $(h=A)$, which is consistent with the softer $m_{t \bar{t}}$ distribution of the $A t \bar{t}$ process as shown in figure 6 , where the $K$-factor is large at small $m_{t \bar{t}}$ at all energies; see figure 8 . The NLO $K$-factor is smaller than unity for $h=H$ at $\sqrt{s}=1000 \mathrm{GeV}$, because the $m_{t \bar{t}}$ dependent $K$ factor becomes smaller than unity at $m_{t \bar{t}} \geq 650 \mathrm{GeV}$ above which the $m_{t \bar{t}}$ distribution is large; see figure 6.

Finally in table 2, we show the total cross sections for the CP-even $(h=H)$ and CPodd $(h=A)$ limits at LO, NLO with stable top quarks, and after Coulomb resummation including the off-shell top quark effects. Despite the factor of 3 enhancements (see table 1), $\sigma(A t \bar{t})$ remains tiny, $0.013 \mathrm{fb}$ at $500 \mathrm{GeV}$. It grows by a factor of 6 to $0.075 \mathrm{fb}$ at $550 \mathrm{GeV}$, which has a significant impact on our CP-violation search at $e^{+} e^{-}$colliders.

Although the NLO and topponium corrections are quite large, the NNLO effects to the total cross sections are expected to be marginal, since potentially large higher order corrections in the $t \bar{t}$ threshold regions are incorporated by Coulomb summation. QCD 
corrections to the angular distributions or correlations of top quark decay products, which may affect determination on $\xi_{h t t}$, are not studied in this paper. Detailed analysis is desired in the future.

\section{Sensitivity of $e^{+} e^{-} \rightarrow h t \bar{t}$ experiments on $\xi_{h t t}$}

In this section, we study the potential of $e^{+} e^{-} \rightarrow h t \bar{t}$ experiments to discover $\mathrm{CP}$ violation in the top Yukawa coupling at a future linear $e^{+} e^{-}$collider by postulating a perfect detector with no systematic uncertainties. Because the measurement accuracy depends on the total number of produced events that determines the statistical errors, we first estimate the total cross section as a function of the parameters of our effective Lagrangian $\left(\kappa_{h t t}, \xi_{h t t}, \kappa_{h Z Z}\right)$ in subsection 4.1. In the next subsection 4.2, we explain in detail how we can calculate the full differential distribution including both semi-leptonic and hadronic decays of $t$ and $\bar{t}$, that are observable by a perfect detector but not as perfect as capable of distinguishing $\bar{d}(d)$ jets from $u(\bar{u})$ jets. In subsection 4.3, we introduce a very simple estimator $\chi^{2}$ function that measures all differences in the observable distributions between experiment $\left(\kappa_{h t t}^{\mathrm{ex}}, \xi_{h t t}^{\mathrm{ex}}\right)$ and theory $\left(\kappa_{h t t}^{\mathrm{th}}, \xi_{h t t}^{\mathrm{th}}\right)$, and study the potential of rejecting $\xi_{h t t}^{\mathrm{th}}=-\xi_{h t t}^{\mathrm{ex}}$ (observation of CP violation) as a function of $\left|\xi_{h t t}^{\mathrm{ex}}\right|$.

\subsection{Total cross section}

The total cross section of the process $e^{+} e^{-} \rightarrow h t \bar{t}$ depends not only on the parameters of $\left(\kappa_{h t t}, \xi_{h t t}, \kappa_{h Z Z}\right)$ but also on the center-of-mass energy $\sqrt{s}$ and the beam polarization. Since the NLO and topponium formation corrections are obtained only for the SM limit $\left(\kappa_{h t t}, \xi_{h t t}, \kappa_{h Z Z}\right)=(1,0,1)$ and for the purely CP-odd limit $\left(\kappa_{h t t}, \xi_{h t t}, \kappa_{h Z Z}\right)=(1, \pm \pi / 2,0)$ for unpolarized beams at $\sqrt{s}=500 \mathrm{GeV}, 550 \mathrm{GeV}, 1000 \mathrm{GeV}$, we make the following simple parameterization

$$
\begin{aligned}
\sigma_{\alpha}\left(\kappa_{h t t}, \xi_{h t t}, \kappa_{h Z Z}\right)= & \sigma_{H}^{\mathrm{NLO}}\left[A_{\alpha}\left(\kappa_{h t t} \cos \xi_{h t t}\right)^{2}+B_{\alpha}\left(\kappa_{h t t} \cos \xi_{h t t} \kappa_{h Z Z}\right)+C_{\alpha}\left(\kappa_{h Z Z}\right)^{2}\right] \\
& +\sigma_{A}^{\mathrm{NLO}}\left[D_{\alpha}\left(\kappa_{h t t} \sin \xi_{h t t}\right)^{2}\right] \\
& +\sigma_{H}^{\text {topp. }}\left[A_{\alpha}^{\prime}\left(\kappa_{h t t} \cos \xi_{h t t}\right)^{2}+B_{\alpha}^{\prime}\left(\kappa_{h t t} \cos \xi_{h t t} \kappa_{h Z Z}\right)+C_{\alpha}^{\prime}\left(\kappa_{h Z Z}\right)^{2}\right] \\
& +\sigma_{A}^{\text {topp. }}\left[D_{\alpha}^{\prime}\left(\kappa_{h t t} \sin \xi_{h t t}\right)^{2}\right] .
\end{aligned}
$$

Here $\sigma_{H}^{\mathrm{NLO}}$ and $\sigma_{A}^{\mathrm{NLO}}$ are obtained from table 1 , whereas we quote the difference between $\sigma^{\mathrm{NLO}}$ and the total cross section after taking account of Coulomb resummation as the 'topponium' cross section,

$$
\sigma^{\text {topp. }}=\sigma^{\text {with Coulomb sum }}-\sigma^{\mathrm{NLO}} .
$$

Those cross sections values are tabulated in table 3. All the coefficients of our parameterization eq. (4.1), which are tabulated in table 4 , are obtained by using the LO matrix elements as follows. We calculate the total cross sections $\sigma_{L}$ and $\sigma_{R}$, respectively, for purely left-handed $\left(e_{L}\right)$ and right-handed $\left(e_{R}\right)$ beam in the LO for several sets of $\left(\kappa_{h t t}, \xi_{h t t}, \kappa_{h Z Z}\right)$ parameters, and obtain the parametrisation eq. (4.1) with $\sigma_{H}^{\mathrm{LO}}$ and $\sigma_{A}^{\mathrm{LO}}$, $\sigma_{H}^{\text {topp. }}=\sigma_{A}^{\text {topp. }}=0$. We approximate the NLO corrections simply by replacing $\sigma_{H}^{\mathrm{LO}}$ and 


\begin{tabular}{|r|cccc|}
\hline$[\mathrm{GeV}]$ & $\sigma_{H}^{\mathrm{NLO}}$ & $\sigma_{H}^{\text {topp. }}$ & $\sigma_{A}^{\mathrm{NLO}}$ & $\sigma_{A}^{\text {topp. }}$ \\
\hline 500 & 0.44 & 0.17 & 0.0079 & 0.0051 \\
550 & 1.41 & 0.15 & 0.064 & 0.011 \\
1000 & 1.97 & - & 0.45 & - \\
\hline
\end{tabular}

Table 3. $e^{+} e^{-} \rightarrow h t \bar{t}$ cross sections at NLO and for the topponium formation, whose sum gives the total cross section after Coulomb resummation in table 1. $h=H$ for the SM Higgs, $h=A$ for the CP-odd Higgs with $\left(\kappa_{h t t}, \xi_{h t t}, \kappa_{h Z Z}\right)=(1, \pm \pi / 2,0)$.

\begin{tabular}{|rc|cccc|cccc|}
\hline$\sqrt{s} \sqrt{s}$ & & $A_{\alpha}$ & $B_{\alpha}$ & $C_{\alpha}$ & $D_{\alpha}$ & $A_{\alpha}^{\prime}$ & $B_{\alpha}^{\prime}$ & $C_{\alpha}^{\prime}$ & $D_{\alpha}^{\prime}$ \\
\hline \multirow{2}{*}{500} & $e_{L}$ & 2.755 & 0.057 & 0.003 & 2.779 & 2.756 & 0.060 & 0.008 & 2.800 \\
& $e_{R}$ & 1.212 & -0.029 & 0.002 & 1.221 & 1.208 & -0.036 & 0.004 & 1.200 \\
550 & $e_{L}$ & 2.726 & 0.061 & 0.009 & 2.766 & 2.716 & 0.074 & 0.029 & 2.773 \\
& $e_{R}$ & 1.222 & -0.025 & 0.007 & 1.234 & 1.202 & -0.043 & 0.022 & 1.227 \\
1000 & $e_{L}$ & 2.562 & 0.078 & 0.084 & 2.736 & - & - & - & - \\
& $e_{R}$ & 1.199 & 0.013 & 0.064 & 1.264 & - & - & - & - \\
\hline
\end{tabular}

Table 4. $e^{+} e^{-} \rightarrow h t \bar{t}$ cross sections at LO, with NLO and Coulomb-resummation corrections for the pure scalar and pseudoscalar processes at $\sqrt{s}=500 \mathrm{GeV}, 550 \mathrm{GeV}$, and $1000 \mathrm{GeV}$.

$\sigma_{A}^{\mathrm{LO}}$ by $\sigma_{H}^{\mathrm{NLO}}$ and $\sigma_{A}^{\mathrm{NLO}}$, respectively. We note here that this is not accurate, and the NLO corrections should be made separately for the $\left(\kappa_{h t t} \cos \xi_{h t t}\right)^{2}$ and the $\left(\kappa_{h Z Z}\right)^{2}$ term as well as the interference term proportional to $\left(\kappa_{h t t} \cos \xi_{h t t} \kappa_{h Z Z}\right)$ in the future. For the topponium coefficients $\left(A_{\alpha}^{\prime}, B_{\alpha}^{\prime}, C_{\alpha^{\prime}}, D_{\alpha}^{\prime}\right)$, we calculate the LO cross sections at $m_{t \bar{t}}=2 m_{t}+0.1 \mathrm{GeV}$, just above the threshold, and fix all the coefficients, normalizing the total cross section to the topponium cross sections obtained in section 3 and tabulated in table 3. Accordingly, the coefficients in table 4 are normalized as

$$
\sum_{\alpha=L, R} A_{\alpha}+B_{\alpha}+C_{\alpha}=\sum_{\alpha=L, R} D_{\alpha}=\sum_{\alpha=L, R} A_{\alpha}^{\prime}+B_{\alpha}^{\prime}+C_{\alpha}^{\prime}=\sum_{\alpha=L, R} D_{\alpha}^{\prime}=4
$$

We believe that this is a good approximation to the $S$-wave topponium formation, which dominates the coulomb resummation corrections.

The cross section for partially polarized beams $\left(\left|P_{e}\right|<1\right.$ for $e^{-},\left|P_{\bar{e}}\right|<1$ for $\left.e^{+}\right)$is obtained from eq. (4.1) as

$$
\sigma\left(P_{e}, P_{\bar{e}}\right)=\frac{\left(1-P_{e}\right)\left(1+P_{\bar{e}}\right)}{4} \sigma_{L}+\frac{\left(1+P_{e}\right)\left(1-P_{\bar{e}}\right)}{4} \sigma_{R}
$$

and hence the unpolarized cross sections are

$$
\sigma(0,0)=\frac{1}{4}\left(\sigma_{L}+\sigma_{R}\right)
$$

Here, we give a few comments on the impacts of the beam polarization on the $h t \bar{t}$ coupling measurements. We first note from table 4 that the ratio $D_{\alpha} / A_{\alpha}$ and $D_{\alpha}^{\prime} / A_{\alpha}^{\prime}$ are almost the 


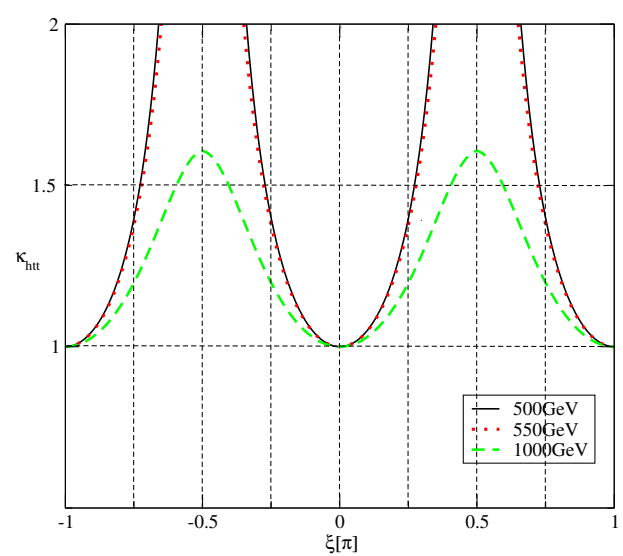

Figure 9. The $\kappa_{h t t}$ value which reproduces the SM $(h=H)$ cross section for $\xi_{h t t} \neq 0$, at $\sqrt{s}=500 \mathrm{GeV}$ (black solid), $550 \mathrm{GeV}$ (red dotted) and $1000 \mathrm{GeV}$ (green dashed). The results are for $\kappa_{h Z Z}=1$.

same for $e_{L}$ and $e_{R}$, and hence the beam polarization has little impacts (besides the total number of events which can be increased by choosing $\left.P_{e}<0<P_{\bar{e}}\right)$ in the measurements of the sign of $\xi_{h t t}$ that arise from the interference between the amplitudes whose squares give $A_{\alpha}$ and $D_{\alpha}$, respectively. The most significant effect of the beam polarization is in the value of $B_{\alpha}$, the coefficients of the interference between two CP-even amplitudes, those with the $h t \bar{t}$ coupling proportional to $\kappa_{h t t} \cos \xi_{h t t}$ and those with the $h Z Z$ coupling. Their signs are opposite at $\sqrt{s}=500 \mathrm{GeV}$ and $550 \mathrm{GeV}$, while the magnitude is different by a factor of six at $\sqrt{s}=1000 \mathrm{GeV}$. Accordingly, we find significant improvement in resolving $\xi_{h t t}=$ $0\left(\cos \xi_{h t t}=1\right)$ and $\xi_{h t t}= \pm \pi\left(\cos \xi_{h t t}=-1\right)$ by using the beam polarization. However, because the beam polarization has little impacts on the CP-violation measurements, the following studies are performed for unpolarized beams $\left(P_{e}=P_{\bar{e}}=0\right)$. We would like to study the impacts of the $e^{+} e^{-}$beam polarizations in a separate report, in which the sensitivity should be compared with those from single top and $h$ production at the LHC [11].

Throughout this study, we adopt

$$
L=1000 \mathrm{fb}^{-1}
$$

as a nominal integrated luminosity at each colliding $e^{+} e^{-}$energy $\sqrt{s}=500 \mathrm{GeV}, 550 \mathrm{GeV}$, and $1000 \mathrm{GeV}$ so that we can compare the impacts of increasing the beam energy. For unpolarized beams, we expect the following number of $h t \bar{t}$ events with unpolarized beams for the SM $(h=H)$ :

$$
\begin{aligned}
& 610 \text { events at } \sqrt{s}=500 \mathrm{GeV} \\
& 1530 \text { events at } \sqrt{s}=550 \mathrm{GeV} \\
& 2000 \text { events at } \sqrt{s}=1000 \mathrm{GeV}
\end{aligned}
$$

which measures the total cross section with statistical errors of $4.0 \%, 2.6 \%, 2.2 \%$, respectively. If we assume that these number of events are observed, then at each energy, the value of $\left(\kappa_{h t t}, \xi_{h t t}\right)$ are constrained to lie on the curves shown in figure 9 . We set $\kappa_{h Z Z}=1$ 
throughout our studies. The statistical error on $\kappa_{h t t}$ is about half the cross section error, $\sim 2.0 \%, \sim 1.3 \%, \sim 1.1 \%$, respectively, for $\sqrt{s}=500 \mathrm{GeV}, 550 \mathrm{GeV}, 1000 \mathrm{GeV}$, which are too small to show in the plot. It is clear that the measurement of the total cross section at two different energies can constrain a region of $\kappa_{h t t}$ and $\xi_{h t t}$, and more importantly, the LHC experiments on $h t \bar{t}$ and $h t, h \bar{t}$ productions will also give such constraints. We therefore assume that the SM Higgs cross section is observed at a given $e^{+} e^{-}$collision energy, and study the capability of distribution studies to resolve the sign of $\xi_{h t t}$ as a function of $\left|\xi_{h t t}\right|$. Therefore, one should be careful when comparing $\xi_{h t t}=\frac{\pi}{4}$ against $\xi_{h t t}=-\frac{\pi}{4}$ at $\sqrt{s}=500 \mathrm{GeV}$, since it is not $\kappa_{h t t}=1$ but $\kappa_{h t t}=1.4$ which gives the same number of events as in the SM.

\subsection{Differential cross sections}

The differential cross sections including $t$ and $\bar{t}$ decay distributions are obtained as in eqs. (2.40) by using the density matrix formalism. In this subsection we explain how we treat the hadronic decays of $t$ and $\bar{t}$, QCD corrections to the differential cross sections which strongly depend on $m_{t \bar{t}}$, possible beam polarizations, and how we take account of the probability that $t$ and $\bar{t}$ cannot be distinguished uniquely when both of them decay hadronically.

First, we introduce the following generic form of the $t$ and $\bar{t}$ decay density matrices

$$
\begin{aligned}
& \rho=\frac{6(1-\bar{x})}{\left(1-\frac{m_{W}^{2}}{m_{t}^{2}}\right)\left(1+2 \frac{m_{W}^{2}}{m_{t}^{2}}\right)}\left(\begin{array}{cc}
\bar{x}+\bar{x}_{z} & \bar{x}_{x}+i \bar{x}_{y} \\
\bar{x}_{x}-i \bar{x}_{y} & \bar{x}-\bar{x}_{z}
\end{array}\right), \\
& \bar{\rho}=\frac{6(1-x)}{\left(1-\frac{m_{W}^{2}}{m_{t}^{2}}\right)\left(1+2 \frac{m_{W}^{2}}{m_{t}^{2}}\right)}\left(\begin{array}{cc}
x+x_{z} & x_{x}-i x_{y} \\
x_{x}+i x_{y} & x-x_{z}
\end{array}\right),
\end{aligned}
$$

respectively, where

$$
\begin{aligned}
\bar{x}^{\mu} & =\frac{2 p_{\bar{\ell}}^{\mu}}{m_{t}}=\left(\bar{x}, \bar{x}_{x}, \bar{x}_{y}, \bar{x}_{z}\right), \\
x^{\mu} & =\frac{2 p_{\ell}^{\mu}}{m_{t}}=\left(x, x_{x}, x_{y}, x_{z}\right),
\end{aligned}
$$

are normalized 4-momenta of $\bar{\ell}(\bar{d}$ or $\bar{s})$ and $\ell(d$ or $s)$, respectively, in the $t$ and $\bar{t}$ rest frame. They are expressed in terms of the $b(\bar{b})$ angles $\theta_{b}^{*}, \phi_{b}^{*}\left(\theta_{\bar{b}}, \bar{\phi}_{\bar{b}}^{*}\right)$ in the $t(\bar{t})$ rest frame, the $\bar{\ell}, \bar{d}, \bar{s}$ angles $\bar{\theta}^{* *}, \bar{\phi}^{* *}$ in the $W^{+}$rest frame, and the $\ell, d, s$ angles $\theta^{* *}, \phi^{* *}$ in the $W^{-}$rest frame. Details are given in appendix A. The density matrices in eq. (4.8) are normalized as

$$
\begin{aligned}
& \int \rho_{\sigma \sigma^{\prime}} \frac{d \cos \theta_{b}^{*} d \phi_{b}^{*}}{4 \pi} \frac{d \cos \bar{\theta}^{* *} d \bar{\phi}^{* *}}{4 \pi}=\delta_{\sigma \sigma^{\prime}} \\
& \int \bar{\rho}_{\bar{\sigma} \bar{\sigma}^{\prime}} \frac{d \cos \theta_{\bar{b}}^{*} d \phi_{\bar{b}}^{*}}{4 \pi} \frac{d \cos \theta^{* *} d \phi^{* *}}{4 \pi}=\delta_{\bar{\sigma} \bar{\sigma}^{\prime}}
\end{aligned}
$$

We use the density matrices eq. (4.8) for all leptonic decays. Indeed, integration of eq. (4.8) over the $b(\bar{b})$ angles for fixed angles of $\bar{\ell}(\ell)$ in the $t(\bar{t})$ rest frame reproduce the well known formula eq. (2.42) for $B_{\ell}=1$. 
For hadronic decays, we introduce the probability $P_{\bar{d} u}$ with which we identify $\bar{d}$ from $u(d$ from $\bar{u})$, or $\bar{s}$ from $c(s$ from $\bar{c})$ in $W^{-}\left(W^{+}\right)$decays

$$
\begin{aligned}
& \rho^{h}=\frac{1+P_{\bar{d} u}}{2} \rho\left(\theta_{b}^{*}, \phi_{b}^{*}, \bar{\theta}^{* *}, \bar{\phi}^{* *}\right)+\frac{1-P_{\bar{d} u}}{2} \rho\left(\theta_{b}^{*}, \phi_{b}^{*}, \pi-\bar{\theta}^{* *}, \pi+\bar{\phi}^{* *}\right) \\
& \bar{\rho}^{h}=\frac{1+P_{\bar{d} u}}{2} \bar{\rho}\left(\theta_{\bar{b}}^{*}, \phi_{\bar{b}}^{*}, \theta^{* *}, \phi^{* *}\right)+\frac{1-P_{\bar{d} u}}{2} \rho\left(\theta_{\bar{b}}^{*}, \phi_{\bar{b}}^{*}, \pi-\theta^{* *}, \pi+\phi^{* *}\right)
\end{aligned}
$$

where we assume that $b$ and $\bar{b}$ momenta are uniquely identified. With the probability $\left(1+P_{\bar{d} u}\right) / 2$, we identify $\bar{d}$ or $\bar{s}(d$ or $s)$ correctly in $W^{+}\left(W^{-}\right)$decays, whereas with the probability $\left(1-P_{\bar{d} u}\right) / 2$, we misidentify $u$ or $s(\bar{u}$ or $\bar{s})$ for $\bar{d}$ or $\bar{s}(d$ or $s)$. In our analysis, we set

$$
P_{\bar{d} u}=P_{\bar{s} c}=0
$$

for simplicity, but $P_{\bar{s} c}$ may be significant. The decay density matrices $\rho^{h}$ and $\bar{\rho}^{h}$ in eqs. (4.11) keep the maximum information given by the matrix elements. If we ignore the top spin sensitivity in the $\bar{d}$ or $\bar{s}(d$ or $s)$ distributions by integrating over the $W$ decay angles $\left(\bar{\theta}^{* *}, \bar{\phi}^{* *}\right)$, only poor resolution power of $b$ angular variables $\theta_{b}^{*}, \phi_{b}^{*}\left(\theta_{\bar{b}}^{*}, \phi_{\bar{b}}^{*}\right)$ remains.

By using $\rho$ and $\bar{\rho}$ in eq. (4.8) for leptonic decays and $\rho^{h}$ and $\bar{\rho}^{h}$ in eq. (4.11) for hadronic decays, the differential cross sections are expressed as follows

$$
\begin{array}{r}
\frac{d \sigma}{d \Phi}=\sum_{\sigma} \sum_{\bar{\sigma}} \sum_{\sigma^{\prime}} \sum_{\bar{\sigma}^{\prime}}\left\{\mathcal{S}_{\sigma \bar{\sigma} \sigma^{\prime} \bar{\sigma}^{\prime}}\left[B_{\ell}^{2} \rho_{\sigma \sigma^{\prime}} \bar{\rho}_{\bar{\sigma} \bar{\sigma}^{\prime}}+B_{\ell} B_{h}\left(\rho_{\sigma \sigma^{\prime}} \bar{\rho}_{\bar{\sigma} \bar{\sigma}^{\prime}}^{h}+\rho_{\sigma \sigma^{\prime}}^{h} \bar{\rho}_{\bar{\sigma} \bar{\sigma}^{\prime}}\right)\right]\right. \\
\left.+B_{h}^{2} \mathcal{S}_{\sigma \bar{\sigma} \sigma^{\prime} \bar{\sigma}^{\prime}}^{h h} \rho_{\sigma \sigma^{\prime}}^{h^{\prime}} \bar{\rho}_{\bar{\sigma} \bar{\sigma}^{\prime}}^{h^{\prime}}\right\}
\end{array}
$$

with the reduced phase space

$$
d \Phi=d \Phi_{h t t} \frac{d \cos \theta_{b}^{*} d \phi_{b}^{*}}{4 \pi} \frac{d \cos \bar{\theta}^{* *} d \bar{\phi}^{* *}}{4 \pi} \frac{d \cos \theta_{\bar{b}}^{*} d \phi_{\bar{b}}^{*}}{4 \pi} \frac{d \cos \theta^{* *} d \phi^{* *}}{4 \pi} .
$$

In this report, we do not distinguish lepton or quark flavours, and adopt

$$
\begin{aligned}
B_{\ell} & =\sum_{\ell=e, \mu, \tau} B\left(t \rightarrow b \bar{\ell} \nu_{\ell}\right) \simeq 0.33 \\
B_{h} & =B(t \rightarrow b \bar{d} u)+B(t \rightarrow b \bar{s} c)=1-B_{\ell} \simeq 0.67 .
\end{aligned}
$$

Production density matrices are expressed in terms of the helicity amplitudes as

$$
\mathcal{S}_{\sigma \bar{\sigma} \sigma^{\prime} \bar{\sigma}^{\prime}}=\frac{1}{2 s} \sum_{\alpha} \frac{1+\alpha P_{e}}{2} \frac{1-\alpha P_{\bar{e}}}{2} M_{\alpha \sigma \bar{\sigma}} M_{\alpha \sigma^{\prime} \bar{\sigma}^{\prime}}^{*}
$$

where $-1<P_{e}, P_{\bar{e}}<1$ denote $e$ and $\bar{e}$ longitudinal beam polarizations.

There is one subtlety when both $t$ and $\bar{t}$ decay hadronically. In this case, identification of $t$ and $\bar{t}$ can be ambiguous, and we introduce the probability $P_{t \bar{t}}$ with which $t$ and $\bar{t}$ can be identified correctly. In this report, we adopt

$$
P_{t \bar{t}}=0.4
$$


which is approximately twice the semi-leptonic decay branching fraction of $B$ mesons. Charge discrimination of hadronic jets from $W^{+}$and $W^{-}$decays may also help. With this probability, the distribution proportional to $B_{h}^{2} \simeq 0.45$ in eq. (4.13) should be

$$
\begin{aligned}
\mathcal{S}_{\sigma \bar{\sigma} \sigma^{\prime} \bar{\sigma}^{\prime}}^{h} \rho_{\sigma \sigma^{\prime}}^{h^{\prime}} \bar{\rho}_{\bar{\sigma} \bar{\sigma}^{\prime}}^{h^{\prime}}= & \frac{1+P_{t \bar{t}}}{2} \mathcal{S}_{\sigma \bar{\sigma} \sigma^{\prime} \bar{\sigma}^{\prime}}(\hat{\theta}, \hat{\phi}) \rho_{\sigma \sigma^{\prime}}^{h}\left(\theta_{b}^{*}, \phi_{b}^{*}, \bar{\theta}^{* *}, \bar{\phi}^{* *}\right) \bar{\rho}_{\bar{\sigma} \bar{\sigma}^{\prime}}^{h}\left(\theta_{\bar{b}}^{*}, \phi_{\bar{b}}^{*}, \theta^{* *}, \phi^{* *}\right) \\
& +\frac{1-P_{t \bar{t}}}{2} \mathcal{S}_{\sigma \bar{\sigma} \sigma^{\prime} \bar{\sigma}^{\prime}}(\pi-\hat{\theta}, \pi+\hat{\phi}) \rho_{\sigma \sigma^{\prime}}^{h}\left(\theta_{\bar{b}}^{*}, \phi_{\bar{b}}^{*}, \theta^{* *}, \phi^{* *}\right) \bar{\rho}_{\bar{\sigma} \bar{\sigma}^{\prime}}^{h}\left(\theta_{b}^{*}, \phi_{b}^{*}, \bar{\theta}^{* *}, \bar{\phi}^{* *}\right)
\end{aligned}
$$

In the last term with the probability $\left(1-P_{t \bar{t}}\right) / 2, t$ and $\bar{t}$ are misidentified, not only the $t$ angles $(\hat{\theta}, \hat{\phi})$ in the $t \bar{t}$ rest frame are replaced by those of $\bar{t}$ angles $(\pi-\hat{\theta}, \pi+\hat{\phi})$, but also all the 4 -angles of $t$ and $\bar{t}$ decays are exchanged. Although this looks complicated, it is straightforward to implement it in a numerical program, and we can keep the maximum surviving information of the matrix elements.

Finally, we implement the perturbative NLO corrections and the topponium contribution as follows. For the NLO corrections, we find in section 3 that the $m_{t \bar{t}}$-dependent $K$-factors are almost identical to the CP-even and CP-odd cases, see figure 8 . We therefore ignore their small differences, and multiply the $m_{t \bar{t}}$-dependent $K$-factor of the CP-even $(h=H)$ cross section to our differential cross section in eq. (4.13)

$$
\frac{d \sigma}{d \Phi} \rightarrow K\left(m_{t \bar{t}}\right) \frac{d \sigma}{d \Phi} .
$$

We confirm that this simple prescription reproduces all the NLO cross sections listed in table 3 .

For the topponium contribution at $\sqrt{s}=500 \mathrm{GeV}$ and $550 \mathrm{GeV}$, we evaluate the differential cross section eq. (4.13) at $m_{t \bar{t}}=2 m_{t}+0.1 \mathrm{GeV}$, and normalize the total integral such that it agrees with the production cross section of topponium + Higgs listed in table 2, by multiplying the factor

$$
\frac{\sigma_{H}^{\text {topp. }}}{\sigma\left(m_{t \bar{t}}=2 m_{t}+0.1 \mathrm{GeV}\right)} .
$$

With this prescription, we obtain all the correlated decays of both $t$ and $\bar{t}$ decays, reproducing the results of ref. [16].

Sophisticated simulation program with topponium formation and decays is needed to evaluate the cross sections and distributions for the SM and its extensions. We believe, however, that the model dependences are approximated reasonably well by using the leading-order matrix elements as outlined in this subsection.

\subsection{Results}

As an estimator for the sensitivity of our CP-violation measurement in $e^{+} e^{-} \rightarrow h t \bar{t}$ process, we introduce the following $\chi^{2}$ function

$$
\chi^{2}\left(\kappa_{h t t}, \xi_{h t t}\right)=L \int d \Phi\left(\frac{d \sigma_{e x} / d \Phi-d \sigma_{t h}\left(\kappa_{h t t}, \xi_{h t t}\right) / d \Phi}{\sqrt{d \sigma_{t h}\left(\kappa_{h t t}, \xi_{h t t}\right) / d \Phi}}\right)^{2},
$$



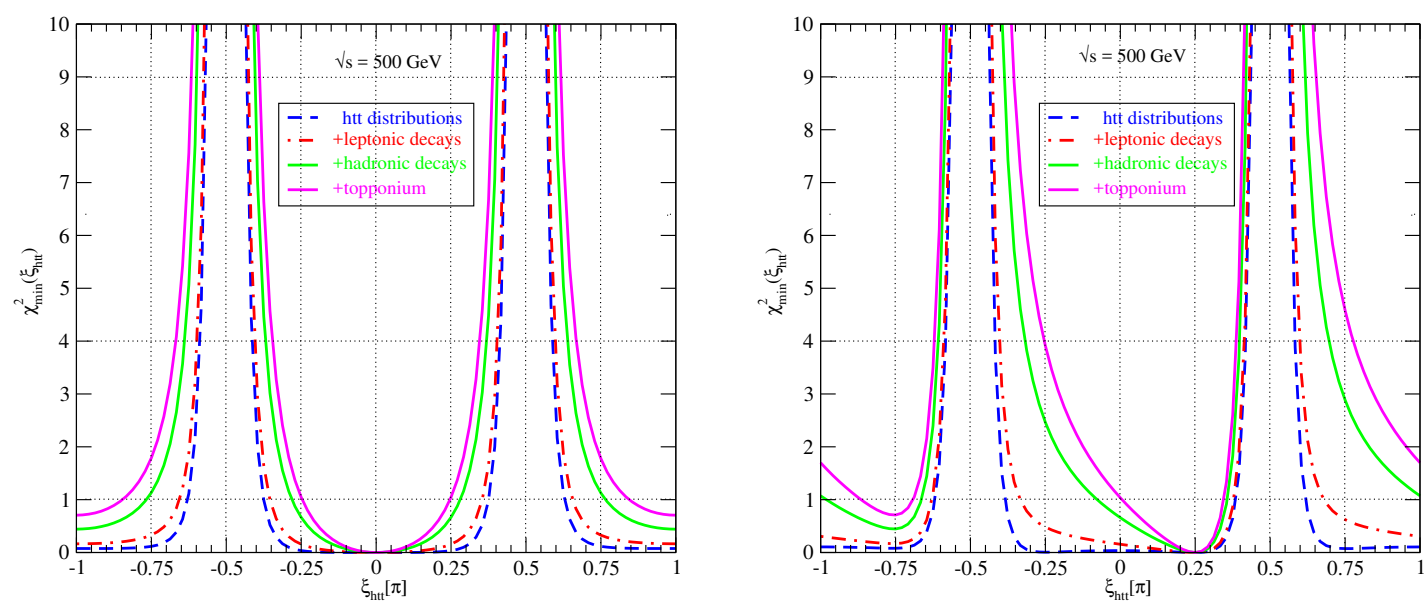

Figure 10. The $\chi^{2}$ plot at $\sqrt{s}=500 \mathrm{GeV}$. $\xi_{h t t}^{\mathrm{ex}}=0$ for the left plot, $\xi_{h t t}^{e x}=\pi / 4$ for the right plot.

Here $d \sigma_{e x} / d \Phi$ represents the observed experimental cross section calculated by assuming that a set of parameters $\left(\kappa_{h t t}^{\mathrm{ex}}, \xi_{h t t}^{\mathrm{ex}}\right)$ are true and $d \sigma_{t h} / d \Phi$ is calculated for an arbitrary set of $\left(\kappa_{h t t}, \xi_{h t t}\right)$ values at each energy. $L$ is the integrated luminosity of the process for unpolarized beams. Throughout our study, we set

$$
L=1000 \mathrm{fb}^{-1}, \quad P_{e}=P_{\bar{e}}=0,
$$

at all energies. The $\chi^{2}$ function in eq. (4.21) accounts for all possible difference between data $d \sigma_{e x} / d \Phi$ and theory $d \sigma_{t h} / d \Phi$ at all kinematical configuration $\Phi$, with the corresponding statistical error proportional to $\left(L d \sigma_{\mathrm{th}} / d \Phi\right)^{-1 / 2}$. It maybe regarded as an ultimate possible sensitivity for a perfect detector with infinite resolution and no errors. Because we calculate the data $d \sigma / d \Phi$ by using our theoretical formula without fluctuations, it is obvious that

$$
\chi_{\min }^{2}=\chi^{2}\left(\kappa_{h t t}^{e x}, \xi_{h t t}^{e x}\right)=0
$$

When we fix the test value of $\xi_{h t t}$, the minimum becomes

$$
\chi_{\min }^{2}\left(\xi_{h t t}\right)=\chi^{2}\left(\kappa_{h t t}\left(\xi_{h t t}\right), \xi_{h t t}\right)
$$

where $\kappa_{h t t}\left(\xi_{h t t}\right)$ is the value which gives the same total cross section with the SM. The trajectory of $\kappa_{h t t}\left(\xi_{h t t}\right)$ has been given in figure 9 for $\sqrt{s}=500 \mathrm{GeV}, 550 \mathrm{GeV}$, and $1000 \mathrm{GeV}$.

In the left panel of figure 10, we show $\chi_{\min }^{2}\left(\xi_{h t t}\right)$ as a function of $\xi_{h t t}$ when the SM distribution $\left(\kappa_{h t t}, \xi_{h t t}\right)=(1,0)$ is assumed for $d \sigma_{e x} / d \Phi$. The blue dashed curve is obtained when only the $h t \bar{t}$ distributions are measured. In our differential cross section formula in eq. (4.19) with $K\left(m_{t \bar{t}}\right)$ for NLO correction, these limits are obtained simply by replacing all our decay density matrices by a unit matrix:

$$
\rho_{\sigma \sigma^{\prime}}=\rho_{\sigma \sigma^{\prime}}^{h}=\delta_{\sigma \sigma^{\prime}}, \quad \bar{\rho}_{\bar{\sigma} \bar{\sigma}^{\prime}}=\bar{\rho}_{\bar{\sigma} \bar{\sigma}^{\prime}}^{h}=\delta_{\bar{\sigma} \bar{\sigma}^{\prime}} .
$$

As compared to the simple calculation of $e^{+} e^{-} \rightarrow h t \bar{t}$ differential cross section, we find slightly smaller $\chi_{\min }^{2}$ because of finite $t \bar{t}$ discrimination probability $P_{t \bar{t}}=0.4$ when both $t$ 
and $\bar{t}$ decay hadronically. The red dash-dotted curve shows our results when leptonic decay angular correlations are assumed. This limit is obtained in our $d \sigma / d \Phi$ formula by setting only the hadronic decay density matrix to be a unit matrix.

$$
\rho_{\sigma \sigma^{\prime}}^{h}=\delta_{\sigma \sigma^{\prime}}, \bar{\rho}_{\bar{\sigma} \bar{\sigma}^{\prime}}^{h}=\delta_{\bar{\sigma} \bar{\sigma}^{\prime}}
$$

Only small improvements are found over the $h t \bar{t}$ distributions only case. This is mainly because of the smallness of the branching fraction factor of the dilepton case $B_{\ell}^{2} \sim 0.11$ even including the $\ell=\tau$ and $\ell=\bar{\tau}$ modes, even though they give full $t$ and $\bar{t}$ decay angular correlations. It is also because the single $(t$ or $\bar{t})$ decay angular distributions with the probability of $2 B_{\ell}\left(1-B_{\ell}\right) \sim 0.44$ have relatively small sensitivity to the CP phase, $\xi_{h t t}$. The green solid curve is obtained by including both leptonic and hadronic decay angular correlations by using our full differential cross sections $d \sigma / d \Phi$ in eq. (4.19). The significant improvement over the red dash-dotted curve shows that the angular correlation between semi-leptonic and hadronic decays keep the resolving power even with the assumption of $P_{\bar{d} u}=P_{\bar{s} c}=0$ in eq. (4.12). Finally, the red solid curve is obtained by adding the topponium contributions. Although the full decay angular distribution studies based on our formalism improve the measurement significantly, it is clear from the plot that the SM data at $\sqrt{s}=500 \mathrm{GeV}$ is consistent with $\left|\xi_{h t t}\right|=\pi / 4$ at $1 \sigma$, even with $1000 \mathrm{fb}^{-1}$.

In the right panel of figure 10, we show the results when the data follow the prediction of the maximum CP phase at $\left(\kappa_{h t t}^{e x}, \xi_{h t t}^{e x}\right)=(1.4, \pi / 4)$, where the total cross section is the same as the SM. Here the $\chi_{\min }^{2}$ value at $\xi_{h t t}=-\xi_{h t t}^{\mathrm{ex}}=-\pi / 4$ gives the sensitivity to CP violation. Apparently, the $h t \bar{t}$ distribution has no sensitivity (see the blue dashed curve), as may be expected from figure 4 in section II, where the difference between $\xi_{h t t}=\pi / 4$ and $-\pi / 4$ is tiny for the sum of squared of all the amplitudes. The inclusion of leptonic decays improves (red dash-dotted line), and the impact of including hadronic decays (green solid line) is quite significant. Adding the topponium contributions, the $\chi^{2}$ value reaches 4 , or we might find a $2 \sigma$ hint of $\mathrm{CP}$ violation.

We find the results discouraging, because this result is for the largest possible CP phase, with $1000 \mathrm{fb}^{-1}$, and a prefect detector with no fluctuations in data are assumed in the analysis. This leads us to re-examine the target energy of a linear collider. As explained in section 2 , the disappointing results at $\sqrt{s}=500 \mathrm{GeV}$ are probably unavoidable, because the small ratio $R=\sigma(A t \bar{t}) / \sigma(H t \bar{t})=0.016$ at $\sqrt{s}=500 \mathrm{GeV}$ shown in figure 5 implies that the CP-odd amplitudes are much smaller than the CP-even amplitudes. Our analytic form of the helicity amplitudes obtained in section II in eq. (2.18) and eqs. (2.21)-(2.26) confirms that all the amplitudes proportional to $\sin \xi_{h t t}$ are either proportional to $\hat{\beta}$, the $t$ velocity in the $t \bar{t}$ rest frame, $\beta$, the velocity of the $t \bar{t}$ system in the $e \bar{e}$ rest frame, or $D_{t}^{1}-D_{t}^{2}$, the difference between the two top quark propagator factors, which are all strongly suppressed at energies near $h t \bar{t}$ threshold, $m_{h}+2 m_{t}=471 \mathrm{GeV}$.

We therefore examine the possibility of upgrading the target energy by $10 \%$ to $550 \mathrm{GeV}$, where the ratio $R=\sigma(A t \bar{t}) / \sigma(H t \bar{t})$ increases to $0.047 \sim(0.22)^{2}$. The results are shown in figure 11. In the left panel, we show $\chi^{2}\left(\xi_{h t t}\right)$ when the $\operatorname{SM}\left(\kappa_{h t t}^{\mathrm{ex}}, \xi_{h t t}^{\mathrm{ex}}\right)=(1,0)$ is assumed for the data. Again, the $h t \bar{t}$ distributions (modified for the probability $B_{h}^{2}\left(1-P_{t \bar{t}}\right)=0.27$ that $t$ and $\bar{t}$ cannot be resolved) shown by blue dashed curve give little sensitivity to $\xi_{h t t}$ 

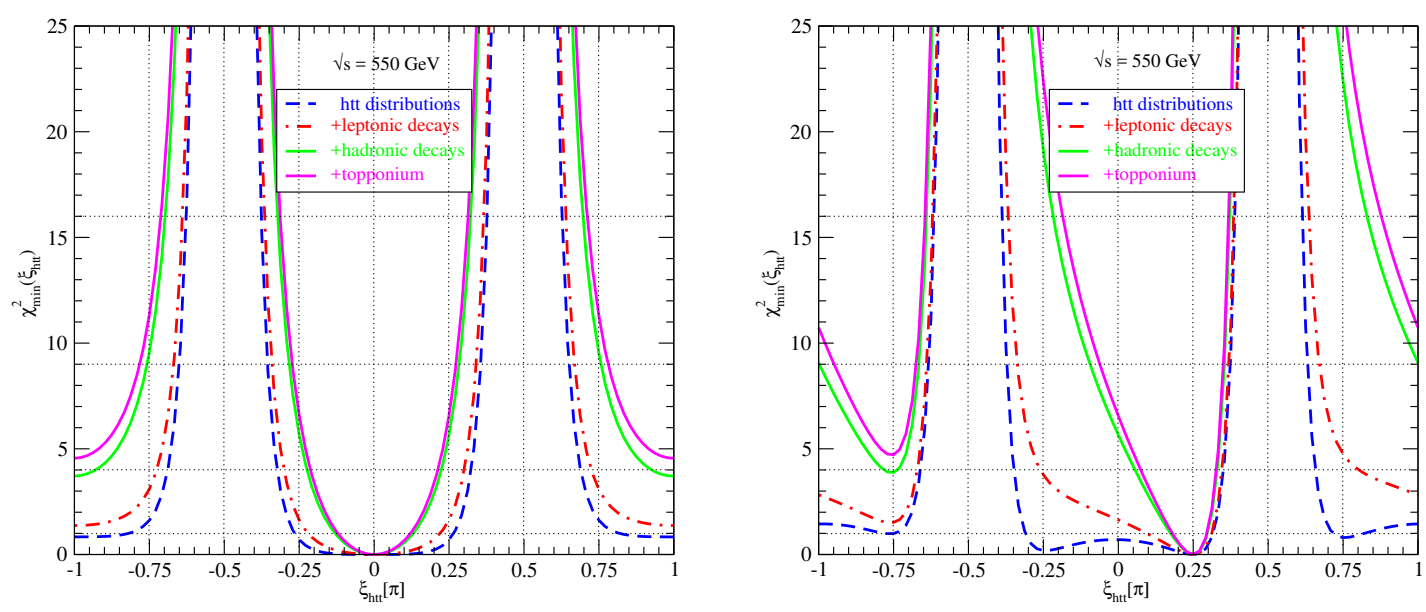

Figure 11. The $\chi^{2}$ plot at $\sqrt{s}=550 \mathrm{GeV}$ assuming $\xi_{h t t}^{e x}=0$ as the observed data (left panel) and $\xi_{h t t}^{e x}=\pi / 4$ as the observed data (right panel).

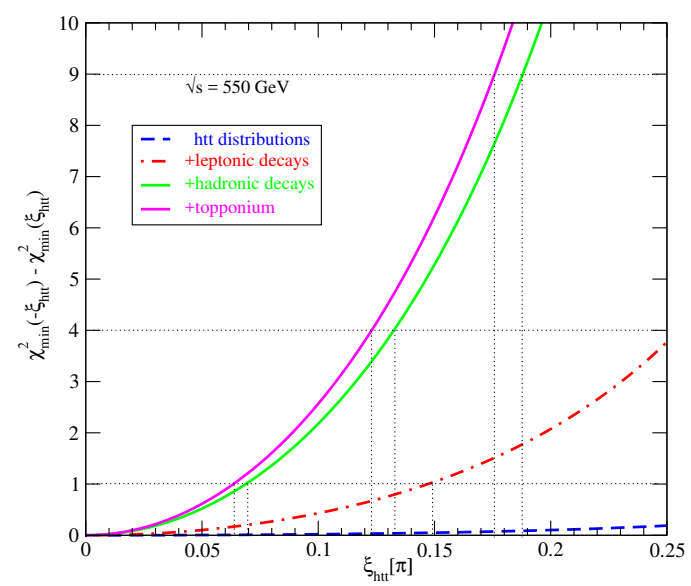

Figure 12. Sensitivity to CP violation is shown by $\chi_{\min }^{2}\left(\xi_{h t t}=-\xi_{h t t}^{\mathrm{ex}}\right)-\chi_{\min }^{2}\left(\xi_{h t t}=\xi_{h t t}^{\mathrm{ex}}\right)$ plotted against $\xi_{h t t}^{\mathrm{ex}}$, the assumed true value of the $\mathrm{CP}$ phase at $\sqrt{s}=550 \mathrm{GeV}$.

when $\left|\xi_{h t t}\right| \leq \pi / 4$. With the inclusion of $t$ or $\bar{t}$ semi-leptonic decay angular correlations, the sensitivity shown by the red dash-dotted curve shoots above $1 \sigma$ at $\left|\xi_{h t t}\right|=\pi / 4$. Moreover with inclusion of the hadronic decay angular correlations, as shown by green solid curve $\chi^{2}$ grows above $5 \sigma$. The topponium contribution is not very significant as may be expected from the relatively small topponium formation cross section at $\sqrt{s}=550 \mathrm{GeV}$. It may be worth noting that $\xi_{h t t}=0$ and $\pi$ can be resolved at $2 \sigma$ level by using decay angular corrections even without beam polarization.

In the right panel, we show the results for $\left(\kappa_{h t t}^{\mathrm{ex}}, \xi_{\text {htt }}^{\mathrm{ex}}\right)=(1.38, \pi / 4)$. As in the $\sqrt{s}=$ $500 \mathrm{GeV}$ case, $h t \bar{t}$ distribution has almost no power in resolving $\mathrm{CP}$ violation (blue dashed curve). $\chi_{\min }^{2}$ value at $\xi_{h t t}=-\xi_{h t t}^{\text {ex }}=-\pi / 4$ becomes $4(2 \sigma)$ if we study lepton angular correlations shown by red dash-dotted curve. It jumps to above $4 \sigma$ once we include hadronic decay angular corrections. We can now hope for a meaningful measurement of $\mathrm{CP}$ violation in the top Yukawa coupling at $\sqrt{s}=550 \mathrm{GeV}$ with dedicated efforts. 

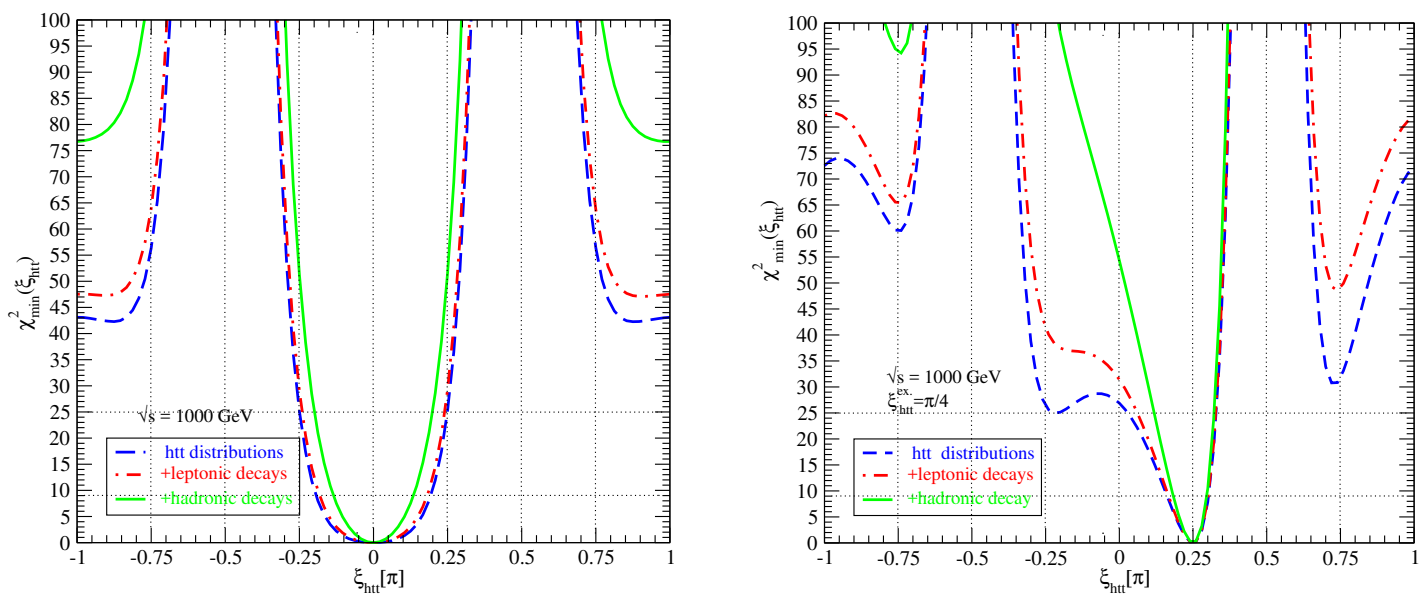

Figure 13. The $\chi^{2}$ plot at $\sqrt{s}=1000 \mathrm{GeV} \cdot \chi_{\min }^{2}\left(\xi_{h t t}\right)$ is plotted against the fit parameter $\xi_{h t t}$, when $\xi_{h t t}^{\mathrm{ex}}=0$ (the SM Higgs) is assumed for the data (left panel) and when $\xi_{h t t}^{\mathrm{ex}}=\pi / 4$ is assumed (right panel).

In figure 12, we show the difference

$$
\chi_{\min }^{2}\left(\xi_{h t t}=-\xi_{h t t}^{\mathrm{ex}}\right)-\chi_{\min }^{2}\left(\xi_{h t t}=\xi_{h t t}^{\mathrm{ex}}\right)
$$

as a function of $\xi_{h t t}^{\mathrm{ex}}$, which gives a measure of the sensitivity of the $e^{+} e^{-} \rightarrow h t \bar{t}$ experiments to $\mathrm{CP}$ violation. We believe that by the time the proposed experiment can be done at the ILC we should have relatively strong constraints on the magnitude of $\xi_{h t t}$ from the $h t \bar{t}$ and single top $+h$ production cross sections and their distributions at the LHC experiments. The role of ILC experiments should hence be to test that there is indeed CP violation in the Higgs Yukawa couplings, when nonzero magnitude of $\xi_{h t t}$ is favored by those data. This can only be done by observing CP violating asymmetries which determine the sign of $\xi_{h t t}$, whose significance is proportional to the difference in eq. (4.27).

In the figure, the blue dashed curve shows that the $h t \bar{t}$ distribution has almost no sensitivity to CP violation. Inclusion of leptonic decay angular correlation (red dash-dotted line) improves, but the most significant improvement is found by including hadronic decay angular correlations as shown by green solid curve. We find that the contributions from the modes where one of $t$ and $\bar{t}$ decays leptonically, and the other decays hadronically, whose branching fractions account for $2 B_{\ell} B_{h} \simeq 0.44$, increase the $\chi_{\min }^{2}$ value most significantly. In this mode, $t$ and $\bar{t}$ are uniquely identified, and the leptonic decay angular distribution is exact. Even though the hadronic decay angular correlations suffer from $\bar{d}$ v.s. $u$ (or $\bar{s}$ v.s. c) misidentification, significant correlation between the $t$ and $\bar{t}$ decays remains to resolve $\mathrm{CP}$ violation. We may tell from figure 12 that a $2 \sigma$ hint of $\mathrm{CP}$ violation can be found if $\xi_{h t t}>0.12 \pi \sim 0.38$, while a $3 \sigma$ evidence of CP violation can be found if $\xi_{h t t}>0.18 \pi \sim 0.55$ at $\sqrt{s}=550 \mathrm{GeV}$ with $L=1000 \mathrm{fb}^{-1}$.

We note in passing that our $\chi^{2}$ function eq. (4.27) adopts the experimental distribution $d \sigma_{\mathrm{ex}} / d \Phi$ calculated analytically for $\xi_{h t t}=\xi_{h t t}^{\mathrm{ex}}$ without fluctuation for realistic binned data. The significance which can be read off from figure 12 should hence be regarded only as a 


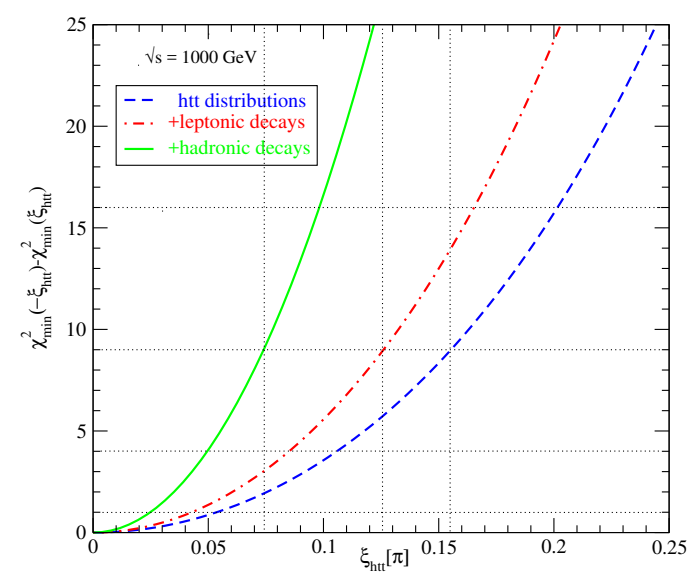

Figure 14. Sensitivity to CP violation is shown by $\chi_{\min }^{2}\left(\xi_{h t t}=-\xi_{h t t}^{\mathrm{ex}}\right)-\chi_{\min }^{2}\left(\xi_{h t t}=\xi_{h t t}^{\mathrm{ex}}\right)$ plotted against $\xi_{h t t}^{\text {ex }}$, the assumed true value of the $\mathrm{CP}$ phase at $\sqrt{s}=1000 \mathrm{GeV}$.

first optimistic estimate. Dedicated studies with event generation may be needed to obtain realistic estimates with statistical fluctuation of experimental data. ${ }^{1}$

We close our discussions by repeating the study at $\sqrt{s}=1000 \mathrm{GeV}$ with $L=1000 \mathrm{fb}^{-1}$. The left panel of figure 13 shows the constraints on $\xi_{h t t}$, when the SM is assumed for the data. At $\sqrt{s}=1000 \mathrm{GeV}$, the $h t \bar{t}$ distribution (blue dashed curve) has significant sensitivity, and it alone can reject $\left|\xi_{h t t}\right|=\pi / 4$ at $5 \sigma$ level. The inclusion of both leptonic and hadronic decay angular correlations (green solid curve) doubles the $\chi_{\min }^{2}$ value. In the right panel, we show the constraint when $\left(\kappa_{h t t}^{\mathrm{ex}}, \xi_{h t t}^{\mathrm{ex}}\right)=(1.2, \pi / 4)$ is assumed for data. $\chi_{\min }^{2}$ value at $\xi_{h t t}=-\xi_{h t t}^{\mathrm{ex}}=-\pi / 4$ tells that $\mathrm{CP}$ violation can be seen at $5 \sigma$ even with $h t \bar{t}$ distribution only (blue dashed curve), while it exceeds $10 \sigma$ once we study full decay angular correlations.

The discovery potential of $\mathrm{CP}$ violation at $\sqrt{s}=1000 \mathrm{GeV}$ with $1000 \mathrm{fb}^{-1}$ is summarized in figure 14. From the green solid curve that is obtained from our full correlation studies, we may hope to find a $3 \sigma$ evidence for CP violation if $\xi_{h t t}^{\mathrm{ex}} \geq 0.074 \pi \sim 0.23$, and a $5 \sigma$ discovery if $\xi_{h t t}^{\mathrm{ex}} \geq 0.12 \pi \sim 0.38$.

\section{Summary and discussions}

In this report, we study the potential of future $e^{+} e^{-}$collider experiments to discover CP violation in the top Yukawa coupling, whose magnitude is largest among all the couplings in the SM. We examine $e^{+} e^{-} \rightarrow h t \bar{t}$ production with all $t$ and $\bar{t}$ decay modes, including both semi-leptonic $(t \rightarrow b \bar{\ell} \nu, \bar{t} \rightarrow \bar{b} \ell \bar{\nu})$ and hadronic decays. The full kinematical distributions including the decay angular correlations are obtained by using the density matrix formalism. In addition to the well known $t$ and $\bar{t}$ decay density matrices for semi-leptonic decays, where the full information on the $t$ and $\bar{t}$ polarization is given by the $\bar{\ell}$ and $\ell$ decay angular distributions in the $t$ and $\bar{t}$ rest frame respectively, we introduce novel decay density matrices for the hadronic decay modes which keep significant $t$ and $\bar{t}$ polarization

\footnotetext{
${ }^{1}$ Significance of $\Delta \chi^{2}$ value obtained by analytically calculated experimental distribution without statistical fluctuation is discussed in e.g. in section 5 of ref. [23].
} 
resolving power even when one cannot distinguish between $\bar{d}$ v.s. $u$ or $\bar{s}$ v.s. $c$ jets $(d$ v.s. $\bar{u}$ or $s$ v.s. $\bar{c}$ jets $)$ in $W^{+}\left(W^{-}\right)$decays.

We approximate the full differential cross section by using the decay angular correlations of the leading-order matrix elements with CP-violating $h t \bar{t}$ coupling phase $\xi_{h t t}$, corrected for the $m_{t \bar{t}}$ dependent NLO correction factor and the topponium formation at $m_{t \bar{t}} \sim 2 m_{t}$. QCD corrections to the angular distributions or correlations of top-quark decay products have not been studied in this paper. Detailed analysis will be required in future work. The sensitivity to $\mathrm{CP}$ violation has been estimated by comparing the full distributions between $\xi_{h t t}$ and $-\xi_{h t t}$ in the range $0 \leq\left|\xi_{h t t}\right|<\pi / 4$, by adjusting the magnitude of the $h t \bar{t}$ coupling $\left(\kappa_{h t t}\right)$ to reproduce the total cross section of the SM Higgs. We study the case at $\sqrt{s}=500 \mathrm{GeV}$ with $\mathcal{L}=1000 \mathrm{fb}^{-1}$, and find that even though the semi-leptonic and hadronic decay angular correlations (as well as topponium contributions) give sensitivity to the sign of $\xi_{h t t}$, it reaches only up to the $2 \sigma$ level after including all the contributions for the maximum CP phase, $\left|\xi_{h t t}\right|=\pi / 4$. We therefore examine the possibility of increasing the beam energy by $10 \%$ to $\sqrt{s}=550 \mathrm{GeV}$. The sensitivity grows significantly, giving us the possibility of a $2 \sigma$ hint for $\left|\xi_{h t t}\right| \sim 0.12 \pi \sim 0.38$ and that of a $3 \sigma$ evidence for $\left|\xi_{h t t}\right| \sim 0.18 \pi \sim 0.55$. We therefore propose that the next target energy of a linear $e^{+} e^{-}$ collider should be increased from the original $500 \mathrm{GeV}$ to $550 \mathrm{GeV}$. Impacts of such a $10 \%$ increase in the beam energy on other key measurements (such as the $h h h$ coupling) should be studied.

At $\sqrt{s}=1000 \mathrm{GeV}$, we expect a $3 \sigma$ evidence at $\left|\xi_{h t t}\right| \sim 0.074 \pi \sim 0.23$, and a $5 \sigma$ discovery at $\left|\xi_{h t t}\right| \sim 0.12 \pi \sim 0.38$. All these numbers should be regarded as possible maximum sensitivity by a perfect detector that can resolve all information given by the matrix elements.

We believe that the $t$ and $\bar{t}$ decay density matrices we obtain for both semi-leptonic and hadronic decay will be a powerful tool in all processes with $t$ and/or $\bar{t}$ in the final states. Their distributions are explained in detail in appendix A. The use of numerical helicity amplitudes evaluated by amplitude calculators can also have wide applications, especially for all the processes whose amplitudes are so complicated that analytic expressions are of little use. We therefore give in appendix B a review of the fermion wave function phase convention in refs. [24-26] that is adopted in MadGraph [27].

\section{A Top and antitop quark decay density matrix}

In this appendix, we give top quark decay density matrices for

$$
t \rightarrow b \bar{\ell} \nu_{\ell}
$$

and

$$
t \rightarrow b \bar{d} u
$$

in the top quark rest frame where the $z$-axis is chosen along the top quark momentum $\vec{p}_{t}$ and the $y$-axis along the $\vec{p}_{t} \times \vec{p}_{h}$ direction in the $t \bar{t}$ rest frame (where the $e^{+} e^{-} \rightarrow h t \bar{t}$ 
helicity amplitudes are calculated). In the frame, we parameterize the four momentum of $b$ and $\bar{\ell}(\bar{d})$ as

$$
\begin{aligned}
p_{b}^{\mu} & =\frac{m_{t}}{2} x_{b}\left(1, \sin \theta_{b}^{*} \cos \phi_{b}^{*}, \sin \theta_{b}^{*} \sin \phi_{b}^{*}, \cos \theta_{b}^{*}\right) \\
p_{\bar{\ell}(\bar{d})}^{\mu} & =\frac{m_{t}}{2} \bar{x}\left(1, \sin \bar{\theta}^{*} \cos \bar{\phi}^{*}, \sin \bar{\theta}^{*} \sin \bar{\phi}^{*}, \cos \bar{\theta}^{*}\right)
\end{aligned}
$$

in the limit of neglecting all the final fermion masses including the $b$-quark.

The decay matrix element for the semileptonic decay (A.1) can be written as

$$
\begin{aligned}
M_{\sigma} & =M\left(t_{\sigma} \rightarrow b \bar{\ell} \nu\right) \\
& =\frac{g^{2}}{2} D_{W}\left(p_{\bar{\ell}}+p_{\nu}\right) u_{L}(b)_{k}^{\dagger}\left(\sigma_{-}^{\mu}\right)_{k \ell} u_{L}(t, \sigma)^{\ell} u_{L}(\nu)_{m}^{\dagger}\left(\sigma_{-\mu}\right)_{m n} v_{L}(\bar{\ell})_{n}
\end{aligned}
$$

with the $W$ propagator factor, $D_{W}(q)=1 /\left(q^{2}-m_{W}^{2}+i m_{W} \Gamma_{W}\right)$, and $\sigma_{ \pm}^{\mu}=(1, \pm \vec{\sigma})$ are the chiral four vectors of the Pauli matrices. By using the Fierz identity

$$
\left(\sigma_{ \pm}^{\mu}\right)_{k \ell}\left(\sigma_{ \pm \mu}\right)_{m n}=2\left(i \sigma^{2}\right)_{k m}\left(i \sigma^{2}\right)_{\ell n}
$$

we can express the amplitudes as

$$
M_{\sigma}=g^{2} D_{W}\left\{u_{L}(b)^{\dagger}\left(i \sigma^{2}\right) u_{L}^{*}(\nu)\right\}\left\{u_{L}(t, \sigma)^{T}\left(i \sigma^{2}\right) v_{L}(\bar{\ell})\right\},
$$

where the bi-fermionic products in each big bracket are separately Lorentz invariant. ${ }^{2}$

The spinor products are calculated easily as

$$
u_{L}(b)^{\dagger}\left(i \sigma^{2}\right) u_{L}^{*}(\nu)=m_{b \nu}=m_{t} \sqrt{1-\bar{x}}
$$

in the $b+\nu$ rest frame, while in the $t$ rest frame we find

$$
u_{L}(t, \sigma)^{T}\left(i \sigma^{2}\right) v_{L}(\bar{\ell})=m_{t}\left(\bar{x} \frac{1+\sigma \cos \bar{\theta}^{*}}{2} e^{i \sigma \bar{\phi}^{*}}\right)^{\frac{1}{2}}
$$

for the top helicity $\sigma / 2$. The amplitudes (A7) are hence simply

$$
M_{\sigma}=g^{2} D_{W} m_{t}^{2}\left(\bar{x}(1-\bar{x}) \frac{1+\sigma \cos \bar{\theta}^{*}}{2} e^{i \sigma \bar{\phi}^{*}}\right)^{\frac{1}{2}} .
$$

We normalize the top quark decay density matrix distribution as

$$
d \rho_{\sigma \sigma^{\prime}}=\frac{1}{2 m_{t} \Gamma_{t}} M_{\sigma} M_{\sigma^{\prime}}^{*} d \Phi_{3}(t \rightarrow b \bar{\ell} \nu)
$$

so that the total integral gives

$$
\int d \rho_{\sigma \sigma^{\prime}}=\frac{1}{2 m_{t} \Gamma_{t}} \int M_{\sigma} M_{\sigma^{\prime}}^{*} d \Phi_{3}(t \rightarrow b \bar{\ell} \nu)=\delta_{\sigma \sigma^{\prime}} B(t \rightarrow b \bar{\ell} \nu)
$$

\footnotetext{
${ }^{2}$ The invariance of each bi-spinors is manifest in the spinor rotation

$$
u_{L}(b)_{\dot{k}}^{*}\left(\sigma_{-}^{\mu}\right)^{\dot{k} \ell} u_{L}(t, \sigma)_{\ell} u^{L}(\nu)_{\dot{m}}^{*}\left(\sigma_{-\mu}\right)^{\dot{m} n} v_{L}(\bar{\ell})_{n}=2 u_{L}(b)_{\dot{k}}^{*} \epsilon^{\dot{k} \dot{m}} u_{L}(\nu)_{\dot{m}}^{*} u_{L}(t, \sigma)_{\ell} \epsilon^{\ell n} v_{L}(\bar{\ell})_{n},
$$

where undotted lower indices give left-handed spinors and the dotted indices are for their complex conjugates. $\epsilon^{k \ell}=\epsilon^{\dot{k} \dot{\ell}}$ are antisymmetric sign factors with $\epsilon_{12}=-\epsilon^{12}=1$.
} 
Note that the trace of (A12) gives twice the branching fraction. Inserting the amplitudes (A10) into (A11), we find

$$
d \rho_{\sigma \sigma^{\prime}}=\frac{g^{4} m_{t}^{4}}{2 m_{t} \Gamma_{t}}\left|D_{W}\right|^{2} \bar{x}(1-\bar{x})\left(\frac{1+\sigma \cos \bar{\theta}^{*}}{2} \frac{1+\sigma^{\prime} \cos \bar{\theta}^{*}}{2}\right)^{\frac{1}{2}} e^{i \frac{\sigma-\sigma^{\prime}}{2} \bar{\phi}^{*}} d \Phi_{3} .
$$

In the case of semi-leptonic decays (A1), it is most convenient to parameterize the invariant 3-body phase space as

$$
d \Phi_{3}=\frac{m_{t}^{2}}{128 \pi^{3}} \bar{x} d \bar{x} \frac{d \cos \bar{\theta}^{*} d \bar{\phi}^{*}}{4 \pi} \frac{d \cos \theta_{b}^{* *}}{2}
$$

where $\theta_{b}^{* *}$ denotes the polar angle of the $b$ quark in the $b+\nu$ rest frame measured from the $-\vec{p}_{\bar{\ell}}$ direction. In the zero width limit of the $W$, we can integrate out $\cos \theta_{b}^{* *}$ as

$$
\begin{aligned}
\int_{-1}^{1} \frac{d \cos \theta_{b}^{* *}}{2}\left|D_{W}\right|^{2} & =\int_{-1}^{1} \frac{d \cos \theta_{b}^{* *}}{2} \frac{\pi}{m_{W} \Gamma_{W}} \delta\left(m_{t}^{2} \bar{x} \frac{1-\cos \theta_{b}^{* *}}{2}-m_{W}^{2}\right) \\
& =\frac{\pi}{m_{W} \Gamma_{W}} \frac{1}{m_{t}^{2} \bar{x}} \theta\left(\bar{x}-\frac{m_{W}^{2}}{m_{t}^{2}}\right) .
\end{aligned}
$$

We integrate out $\bar{x}$ in the region $m_{W}^{2} / m_{t}^{2}<\bar{x}<1$, and find

$$
d \rho_{\sigma \sigma^{\prime}}=B(t \rightarrow b \bar{\ell} \nu)\left(1+\sigma \cos \bar{\theta}^{*}\right)^{\frac{1}{2}}\left(1+\sigma^{\prime} \cos \bar{\theta}^{*}\right)^{\frac{1}{2}} e^{i \frac{\sigma-\sigma^{\prime}}{2} \bar{\phi}^{*}} \frac{d \cos \bar{\theta}^{*} d \bar{\phi}^{*}}{4 \pi} .
$$

In the matrix form

$$
d \rho=B(t \rightarrow b \bar{\ell} \nu)\left(\begin{array}{cc}
1+\cos \bar{\theta}^{*} & \sin \bar{\theta}^{*} e^{i \bar{\phi}^{*}} \\
\sin \bar{\theta}^{*} e^{-i \bar{\phi}^{*}} & 1-\cos \bar{\theta}^{*}
\end{array}\right) \frac{d \cos \bar{\theta}^{*} d \bar{\phi}^{*}}{4 \pi}
$$

reproducing the normalization eq. (A12).

In the case of the hadronic decay (A2) of the top quark, matrix elements are exactly the same as those of the semileptonic decays eq. (A10), where $\left(\bar{x}, \bar{\theta}^{*}, \bar{\phi}^{*}\right)$ are now the normalized energy and the angles of the $\bar{d}$ quark (or $\bar{s}$ in the case of $W \rightarrow \bar{s}$ c decay); see eq. (A4). We need, however, a careful treatment of the decay phase space integral, because it is difficult to identify $\bar{d}$ from $u(\bar{s}$ from $c)$ in collider experiments. We introduce a parameter $P_{\bar{d} u}\left(0 \leq P_{\bar{d} u} \leq 1\right)$, which measures the probability that $\bar{d}$ v.s. $u(\bar{s}$ v.s. $c)$ are distinguished correctly, in the decay density matrix distributions. Although all the results presented in this report are for $P_{\bar{d} u}=P_{\bar{s} c}=0$ (absolutely no distinction between the two decaying jets of the $W$ ), we hope that our formalism encourages further efforts for $\bar{s}$ v.s. $c$ discrimination (or even $\bar{d}$ v.s. $u$ ).

We start with the differential density matrices eq. (A.13), multiplied by a factor of 3 for the color, where $\left(\bar{x}, \bar{\theta}^{*}, \bar{\phi}^{*}\right)$ are parameterizing $\bar{d}(\bar{s})$ quark momentum in the top quark rest frame. Because $\bar{d}$ and $u(\bar{s}$ and $c)$ are difficult to distinguish, we parameterize the $t \rightarrow b \bar{d} u$ phase space by using the $W \rightarrow \bar{d} u$ rest frame angles:

$$
d \Phi_{3}=\frac{1}{64 \pi^{2}}\left(1-\frac{m_{\bar{d} u}^{2}}{m_{t}^{2}}\right) \frac{d \cos \theta_{b}^{*} d \phi_{b}^{*}}{4 \pi} \frac{d m_{\bar{d} u}^{2}}{2 \pi} \frac{d \cos \bar{\theta}^{* *} d \bar{\phi}^{* *}}{4 \pi}
$$


where $\theta_{b}^{*}$ and $\phi_{b}^{*}$ give the $b$ quark momentum in the top quark rest frame, while $\bar{\theta}^{* *}$ and $\bar{\phi}^{* *}$ give the $\bar{d}$ momentum in the $W \rightarrow \bar{d} u$ rest frame which is obtained from the top quark rest frame by rotations $\left(-\phi_{b}^{*}\right.$ about the $z$-axis, and then $-\theta_{b}^{*}$ about the $y$-axis) and a Lorentz boost along the $z$-axis by

$$
\beta=\frac{1-m_{\bar{d} u}^{2} / m_{t}^{2}}{1+m_{\bar{d} u}^{2} / m_{t}^{2}}
$$

The integration over $m_{\bar{d} u}^{2}$ is done in the zero $W$ width limit to obtain

$$
\int_{0}^{m_{t}^{2}} \frac{d m_{\bar{d} u}^{2}}{2 \pi}\left|D_{W}\right|^{2}=\frac{1}{2 m_{W} \Gamma_{W}}
$$

and we find

$$
d \rho_{\sigma \sigma^{\prime}}=\frac{6 B(t \rightarrow b \bar{d} u)}{\left(1-\frac{m_{W}^{2}}{m_{t}^{2}}\right)\left(1+2 \frac{m_{W}^{2}}{m_{t}^{2}}\right)} \hat{\rho}_{\sigma \sigma^{\prime}} \frac{d \cos \theta_{b}^{*} d \phi_{b}^{*}}{4 \pi} \frac{d \cos \bar{\theta}^{* *} d \bar{\phi}^{* *}}{4 \pi}
$$

where

$$
\hat{\rho}=(1-\bar{x})\left(\begin{array}{cc}
\bar{x}+\bar{x}_{z} & \bar{x}_{x}+i \bar{x}_{y} \\
\bar{x}_{x}-i \bar{x}_{y} & \bar{x}-\bar{x}_{z}
\end{array}\right) .
$$

Here, $\bar{x}^{\mu}=\left(\bar{x}, \bar{x}_{x}, \bar{x}_{y}, \bar{x}_{z}\right)$ is the normalized $\bar{d}$ quark four momentum in the top quark rest frame

$$
\begin{aligned}
\bar{x} & =\frac{m_{W}}{m_{t}} \gamma^{*}\left(1-\beta^{*} \cos \bar{\theta}^{* *}\right) \\
\bar{x}_{x} & =\frac{m_{W}}{m_{t}}\left\{\cos \phi_{b}^{*}\left(\cos \theta_{b}^{*} \sin \bar{\theta}^{* *} \cos \bar{\phi}^{* *}+\sin \theta_{b}^{*} \gamma^{*}\left(\cos \bar{\theta}^{* *}-\beta^{*}\right)\right)-\sin \phi_{b}^{*} \sin \bar{\theta}^{* *} \sin \bar{\phi}^{* *}\right\} \\
\bar{x}_{y} & =\frac{m_{W}}{m_{t}}\left\{\sin \phi_{b}^{*}\left(\cos \theta_{b}^{*} \sin \bar{\theta}^{* *} \cos \bar{\phi}^{* *}+\sin \theta_{b}^{*} \gamma^{*}\left(\cos \bar{\theta}^{* *}-\beta^{*}\right)\right)+\cos \phi_{b}^{*} \sin \bar{\theta}^{* *} \sin \bar{\phi}^{* *}\right\} \\
\bar{x}_{z} & =\frac{m_{W}}{m_{t}}\left\{-\sin \theta_{b}^{*} \sin \bar{\theta}^{* *} \cos \bar{\phi}^{* *}+\cos \theta_{b}^{*} \gamma^{*}\left(\cos \bar{\theta}^{* *}-\beta^{*}\right)\right\},
\end{aligned}
$$

where $\gamma^{*}=\frac{m_{t}}{2 m_{W}}\left(1+\frac{m_{W}^{2}}{m_{t}^{2}}\right), \gamma^{*} \beta^{*}=\frac{m_{t}}{2 m_{W}}\left(1-\frac{m_{W}^{2}}{m_{t}^{2}}\right)$. With the above parameterization, we can express the top quark decay density matrix distribution under realistic experimental conditions with finite (or zero) probability $P_{\bar{d} u}$ for $\bar{d}$ v.s. $u(\bar{s}$ v.s. $c$ ) discrimination as follows

$$
\begin{aligned}
d \rho\left(P_{\bar{d} u}\right)= & \frac{6 B(t \rightarrow b \bar{d} u)}{\left(1-\frac{m_{W}^{2}}{m_{t}^{2}}\right)\left(1+2 \frac{m_{W}^{2}}{m_{t}^{2}}\right)} \\
& \times\left\{\frac{1+P_{\bar{d} u}}{2} \hat{\rho}\left(\theta_{b}^{*}, \phi_{b}^{*}, \bar{\theta}^{* *}, \bar{\phi}^{* *}\right)+\frac{1-P_{\bar{d} u}}{2} \hat{\rho}\left(\theta_{b}^{*}, \phi_{b}^{*}, \pi-\bar{\theta}^{* *}, \pi+\bar{\phi}^{* *}\right)\right\} \\
& \times \frac{d \cos \theta_{b}^{*} d \phi_{b}^{*}}{4 \pi} \frac{d \cos \bar{\theta}^{* *} d \bar{\phi}^{* *}}{4 \pi} .
\end{aligned}
$$

The meaning of eq. (A.24) is that, if $\bar{d}$ v.s. $u$ discrimination is perfect, i.e. $P_{\bar{d} u}=1$, then the density matrix distribution is exactly the same (up to the branching fraction factors) as 
that for the semi-leptonic decays (A17). When $P_{d \bar{u}}=0$, which we assume in our numerical studies, the $\bar{d}$ quark (at $\bar{\theta}^{* *}$ and $\bar{\phi}^{* *}$ ) is not distinguished from the $u$ quark (at $\pi-\bar{\theta}^{* *}$ and $\left.\pi+\bar{\phi}^{* *}\right)$ in the $W$ rest frame, and hence their average measures the top quark polarization.

In the case of the $\bar{t}$ decay amplitudes, as compared to eqs. (A.5) and (A.9), we find

$$
\begin{aligned}
\bar{M}_{\bar{\sigma}}\left(\bar{t}_{\bar{\sigma}} \rightarrow \bar{b} \ell \bar{\nu}\right) & =\frac{g^{2}}{2} D_{W}\left(p_{\ell}+p_{\bar{\nu}}\right) v_{L}^{\dagger}(\bar{t}, \bar{\sigma})\left(\sigma_{-}^{\mu}\right) v_{L}(\bar{b}) u_{L}(\ell)^{\dagger}\left(\sigma_{-\mu}\right) v_{L}(\bar{\nu}) \\
& \left.=g^{2} D_{W}\left\{v_{L}^{\dagger}(\bar{t}, \bar{\sigma})\left(i \sigma^{2}\right) u_{L}(\ell)^{*}\right\}\left\{v_{L}(\bar{b})^{T}\left(i \sigma^{2}\right) v_{L}(\bar{\nu})\right)\right\} .
\end{aligned}
$$

We parameterize the $\bar{b}$ and $\ell(d)$ four momenta exactly in the same ways as eqs. (A.3), (A.4) for $t$ decays

$$
\begin{aligned}
p_{\bar{b}}^{\mu} & =\frac{m_{t}}{2} x_{\bar{b}}\left(1, \sin \theta_{\bar{b}}^{*} \cos \phi_{\bar{b}}^{*}, \sin \theta_{\bar{b}}^{*} \sin \phi_{\bar{b}}^{*}, \cos \theta_{\bar{b}}^{*}\right) \\
p_{\ell(d)}^{\mu} & =\frac{m_{t}}{2} x\left(1, \sin \theta^{*} \cos \phi^{*}, \sin \theta^{*} \sin \phi^{*}, \cos \theta^{*}\right)
\end{aligned}
$$

in the $\bar{t}$ rest frame which is obtained from the $t$ rest frame by a Lorentz boost along the $z$-axis. In this way, the azimuthal angles are measured from the common $x$-axis, while the $\bar{t}$ helicity corresponds to its spin component along the negative $z$-axis. In this frame, we find the helicity amplitudes are given as

$$
\bar{M}_{\bar{\sigma}}=g^{2} D_{W} m_{t}^{2}\left(x(1-x) \frac{1+\bar{\sigma} \cos \theta^{*}}{2} e^{-i \bar{\sigma} \phi^{*}}\right)^{\frac{1}{2}} .
$$

Therefore the $\bar{t}$ decay density matrix for the semi-leptonic decays is

$$
d \bar{\rho}=B(\bar{t} \rightarrow \bar{b} \ell \bar{\nu})\left(\begin{array}{cc}
1+\cos \theta^{*} & \sin \theta^{*} e^{-i \phi^{*}} \\
\sin \theta^{*} e^{i \phi^{*}} & 1-\cos \theta^{*}
\end{array}\right) \frac{d \cos \theta^{*} d \phi^{*}}{4 \pi}
$$

and that for the hadronic decays reads

$$
\begin{aligned}
d \bar{\rho}_{\bar{\sigma} \bar{\sigma}^{\prime}} & =\frac{6 B(\bar{t} \rightarrow \bar{b} d \bar{u})}{\left(1-\frac{m_{W}^{2}}{m_{t}^{2}}\right)\left(1+2 \frac{m_{W}^{2}}{m_{t}^{2}}\right)} \hat{\bar{\rho}}_{\bar{\sigma} \bar{\sigma}^{\prime}} \frac{d \cos \theta_{\bar{b}}^{*} d \phi_{\bar{b}}^{*}}{4 \pi} \frac{d \cos \theta^{* *} d \phi^{* *}}{4 \pi} \\
\hat{\bar{\rho}} & =(1-x)\left(\begin{array}{cc}
x+x_{z} & x_{x}-i x_{y} \\
x_{x}+i x_{y} & x-x_{z}
\end{array}\right),
\end{aligned}
$$

if we can distinguish between $d$ and $\bar{u}$ jets. As in eq. (A.23), $x^{\mu}=\left(x, x_{x}, x_{y}, x_{z}\right)$ is the normalized $d$ (or $s$ ) quark four momentum in the $\bar{t}$ rest frame

$$
\begin{aligned}
x & =\frac{m_{W}}{m_{t}} \gamma^{*}\left(1-\beta^{*} \cos \theta^{* *}\right) \\
x_{x} & =\frac{m_{W}}{m_{t}}\left\{\cos \phi_{\bar{b}}^{*}\left(\cos \theta_{\bar{b}}^{*} \sin \theta^{* *} \cos \phi^{* *}+\sin \theta_{\bar{b}}^{*} \gamma^{*}\left(\cos \theta^{* *}-\beta^{*}\right)\right)-\sin \phi_{\bar{b}}^{*} \sin \theta^{* *} \sin \phi^{* *}\right\} \\
x_{y} & =\frac{m_{W}}{m_{t}}\left\{\sin \phi_{\bar{b}}^{*}\left(\cos \theta_{\bar{b}}^{*} \sin \theta^{* *} \cos \phi^{* *}+\sin \theta_{\bar{b}}^{*} \gamma^{*}\left(\cos \theta^{* *}-\beta^{*}\right)\right)+\cos \phi_{\bar{b}}^{*} \sin \theta^{* *} \sin \phi^{* *}\right\} \\
x_{z} & =\frac{m_{W}}{m_{t}}\left\{-\sin \theta_{\bar{b}}^{*} \sin \theta^{* *} \cos \phi^{* *}+\cos \theta_{\bar{b}}^{*} \gamma^{*}\left(\cos \theta^{* *}-\beta^{*}\right)\right\} .
\end{aligned}
$$


With the probability $P_{d \bar{u}}=P_{\bar{d} u}$ for $d$ v.s. $\bar{u}$ discrimination, the $\bar{t}$ decay density matrix for hadronic decays becomes

$$
\begin{aligned}
d \bar{\rho}\left(P_{d \bar{u}}\right)= & \frac{6 B(\bar{t} \rightarrow \bar{b} \bar{d} u)}{\left(1-\frac{m_{W}^{2}}{m_{t}^{2}}\right)\left(1+2 \frac{m_{W}^{2}}{m_{t}^{2}}\right)} \\
& \times\left\{\frac{1+P_{d \bar{u}}}{2} \hat{\bar{\rho}}\left(\theta_{\bar{b}}^{*}, \phi_{\bar{b}}^{*}, \theta^{* *}, \phi^{* *}\right)+\frac{1-P_{d \bar{u}}}{2}\left(\theta_{\bar{b}}^{*}, \phi_{\bar{b}}^{*}, \pi-\theta^{* *}, \pi+\phi^{* *}\right)\right\} \\
& \times \frac{d \cos \theta_{\bar{b}}^{*} d \phi_{\bar{b}}^{*}}{4 \pi} \frac{d \cos \theta^{* *} d \phi^{* *}}{4 \pi} .
\end{aligned}
$$

It is worth noting here that the $t \rightarrow b \bar{\ell} \nu$ and $\bar{t} \rightarrow \bar{b} \ell \bar{\nu}$ amplitudes as shown in eq. (A.10) and eq. (A.29), respectively, are expressed as squared roots of complex numbers because space rotations on spinors give half angles. These elegant expressions cannot be obtained if one adopts a phase convention that depends on the azimuthal angle, such as the one in refs. [24-26] adopted by a Feynman amplitude calculator MadGraph [27]. The amplitudes eq. (A.10) and eq. (A.29) can be recovered by supplying the phase factor in eq. (B.7), as will be explained in detail in appendix B. Because the phase factor associated with the $\bar{\ell}$ and $\ell$ spinors, respectively, for $t$ and $\bar{t}$ decay amplitudes are common for both helicity amplitudes, the decay density matrices in eqs. (A.17), (A.22), (A.30), (A.32) are independent of the $\bar{\ell}$ and $\ell$ spinor phase convention.

\section{B HELAS phase convention for the density matrix}

As explained above, we use HELAS amplitudes as obtained by MadGraph to test our analytic calculations, and to generate differential cross sections with full decay correlations. We encounter a subtle frame dependence of the $h t \bar{t}$ production density matrix elements due to the specific phase convention, hereafter called HZ convention [24], which has been adopted by HELAS subroutines [25, 26]. Because the use of MadGraph-generated HELAS amplitudes can be a powerful tool for studying massive fermion spin correlations in various experiments, we show in this appendix the origin of the frame dependence and ways to avoid possible inconsistencies.

In ref. [24], massive fermion wave functions are expressed in terms of two helicity spinors

$$
\begin{aligned}
& \chi_{+}(\vec{p})=\left(\begin{array}{c}
\cos \frac{\theta}{2} \\
\sin \frac{\theta}{2} e^{i \phi}
\end{array}\right) \\
& \chi_{-}(\vec{p})=\left(\begin{array}{c}
-\sin \frac{\theta}{2} e^{-i \phi} \\
\cos \frac{\theta}{2}
\end{array}\right)
\end{aligned}
$$

when the fermion four momentum is

$$
p^{\mu}=(E, \vec{p})=(E, p \sin \theta \cos \phi, p \sin \theta \sin \phi, p \cos \theta)
$$

where $p=|\vec{p}|, 0 \leq \theta<\pi$ so that $\cos \frac{\theta}{2}, \sin \frac{\theta}{2}>0$ and $0 \leq \phi<2 \pi$. Note that

$$
\begin{aligned}
& \chi_{-}(\vec{p})=-i \sigma^{2} \chi_{+}(\vec{p})^{*} \\
& \chi_{+}(\vec{p})=i \sigma^{2} \chi_{-}(\vec{p})^{*} .
\end{aligned}
$$


By starting from the fermion spinors in the rest frame,

$$
u_{L}(p,+)=u_{R}(p,+)=\sqrt{m}\left(\begin{array}{l}
1 \\
0
\end{array}\right), \quad u_{L}(p,-)=u_{R}(p,-)=\sqrt{m}\left(\begin{array}{l}
0 \\
1
\end{array}\right),
$$

and with the charge conjugation

$$
v_{L}(p, \sigma)=i \sigma^{2} u_{R}(p, \sigma)^{*}, \quad v_{R}(p, \sigma)=-i \sigma^{2} u_{L}(p, \sigma)^{*},
$$

we find that straightforward calculation of the Lorentz transformation, from the $t$ and $(\bar{t})$ rest frame $p^{\mu}=(m, 0,0,0)$ to the frame in which the $t(\bar{t})$ four momentum becomes eq. (B.2),

$$
\begin{aligned}
& u(p,+)=\left(\begin{array}{c}
\sqrt{E-p} \chi_{+}(\vec{p}) \\
\sqrt{E+p} \chi_{+}(\vec{p})
\end{array}\right) e^{-i \frac{\phi}{2}} \\
& u(p,-)=\left(\begin{array}{c}
\sqrt{E+p} \chi_{-}(\vec{p}) \\
\sqrt{E-p} \chi_{-}(\vec{p})
\end{array}\right) e^{i \frac{\phi}{2}} \\
& v(p,+)=\left(\begin{array}{c}
-\sqrt{E+p} \chi_{-}(\vec{p}) \\
\sqrt{E-p} \chi_{-}(\vec{p})
\end{array}\right) e^{i \frac{\phi}{2}} \\
& v(p,-)=\left(\begin{array}{c}
\sqrt{E-p} \chi_{+}(\vec{p}) \\
-\sqrt{E+p} \chi_{+}(\vec{p})
\end{array}\right) e^{-i \frac{\phi}{2}}
\end{aligned}
$$

The fermion wave functions in ref. [24], adopted in HELAS [25, 26] are obtained by chopping off the phase factors which depend on the azimuthal angle and the helicity of $t$ and $\bar{t}$,

$$
\begin{aligned}
& u(p, \sigma)=u(p, \sigma)_{\mathrm{HZ}} e^{-i \frac{\sigma \phi}{2}} \\
& v(p, \bar{\sigma})=v(p, \bar{\sigma})_{\mathrm{HZ}} e^{i \frac{\bar{\sigma} \phi}{2}}
\end{aligned}
$$

Hence, for all $t \bar{t}$ production processes, the helicity amplitudes in the HZ phase convention differ from the naive ones as

$$
M_{\sigma \bar{\sigma}}=\left(M_{\sigma \bar{\sigma}}\right)_{\mathrm{HZ}}\left(e^{i \frac{\sigma \phi+\bar{\sigma} \bar{\phi}}{2}}\right),
$$

when $t$ and $\bar{t}$ momenta have non-zero azimuthal angles, $\phi$ and $\bar{\phi}$, respectively.

In our study, we calculate the helicity amplitudes in the $t \bar{t}$ rest frame where

$$
\bar{\phi}=\phi
$$

holds. ${ }^{3}$ Therefore the helicity amplitudes in HZ convention (the HELAS amplitudes) differ from the conventional amplitudes by the following phase factors

$$
\begin{aligned}
& M_{++}=\left(M_{++}\right)_{\mathrm{HZ}} e^{i \phi} \\
& M_{--}=\left(M_{--}\right)_{\mathrm{HZ}} e^{-i \phi} \\
& M_{ \pm \mp}=\left(M_{ \pm \mp}\right)_{\mathrm{HZ}} \cdot
\end{aligned}
$$

\footnotetext{
${ }^{3}$ In order to avoid purely complex phase $e^{ \pm i \pi / 2}$ for the $\bar{t}$ wave function, we obtain the $\bar{t}$ momentum by setting $\theta_{\bar{t}}=-\pi+\theta_{t}$ and $\phi_{\bar{t}}=\phi_{t}$ in the $t \bar{t}$ rest frame.
} 
Although the phase factors do not affect the magnitudes of each helicity amplitudes, they do not decouple from the off-diagonal elements of the density matrix $\rho_{\sigma \bar{\sigma}, \sigma^{\prime} \bar{\sigma}^{\prime}}=M_{\sigma \bar{\sigma}} M_{\sigma^{\prime} \bar{\sigma}^{\prime}}^{*}$,

$$
\begin{aligned}
& \rho_{++,--}=\left(\rho_{++,--}\right)_{\mathrm{HZ}} e^{i 2 \phi} \\
& \rho_{++, \pm \mp}=\left(\rho_{++, \pm \mp}\right)_{\mathrm{HZ}} e^{i \phi} \\
& \rho_{+-,-+}=\left(\rho_{+-,-+}\right)_{\mathrm{HZ}} .
\end{aligned}
$$

They introduce unphysical $\phi$-dependences in real and imaginary parts of the density matrices, when $t$ and $\bar{t}$ momentum has non-zero azimuthal angles. Note that the phase does not appear in the chirality conserving amplitudes in the massless limit, and hence the $e^{+} e^{-}$ currents are free from the azimuthal angle phases.

Such convention dependent $\phi$-dependence does not survive in the physical distributions. For instance, the phases in eq. (B.11) are precisely canceled by the corresponding phases in the $t$ and $\bar{t}$ decay density matrices, if we use the same wave functions for production and decay. In order to recover the rotational invariance of the conventional helicity amplitudes, we may supply the phase factor in eq. (B.8) or simply stick to the frame where $t$ and $\bar{t}$ momenta have no $y$-components. We adopt in section 2 the latter approach by choosing the frame in which $t$ and $\bar{t}$ momenta are in the $x-z$ plane, whereas the azimuthal angles are given to $e$ and $\bar{e}$ momenta.

\section{Acknowledgments}

The authors wish to thank Pyungwon Ko in KIAS, Tao Han in University of Pittsburgh and Shinya Kanemura in University of Toyama and Osaka University for support where part of this work was done. YZ thanks Kentarou Mawatari for helpful discussions and JSPS for financial support. This work is supported in part by Grant-in-Aid for Scientific Research (No. 16F16321) from JSPS.

Open Access. This article is distributed under the terms of the Creative Commons Attribution License (CC-BY 4.0), which permits any use, distribution and reproduction in any medium, provided the original author(s) and source are credited.

\section{References}

[1] ATLAS collaboration, Measurements of the Higgs boson production and decay rates and coupling strengths using pp collision data at $\sqrt{s}=7$ and $8 \mathrm{TeV}$ in the ATLAS experiment, Eur. Phys. J. C 76 (2016) 6 [arXiv: 1507.04548] [InSPIRE].

[2] ATLAS and CMS collaborations, Measurements of the Higgs boson production and decay rates and constraints on its couplings from a combined ATLAS and CMS analysis of the LHC pp collision data at $\sqrt{s}=7$ and $8 \mathrm{TeV}$, JHEP 08 (2016) 045 [arXiv: 1606. 02266] [INSPIRE].

[3] J.F. Gunion and X.-G. He, Determining the CP nature of a neutral Higgs boson at the LHC, Phys. Rev. Lett. 76 (1996) 4468 [hep-ph/9602226] [INSPIRE].

[4] J. Ellis, D.S. Hwang, K. Sakurai and M. Takeuchi, Disentangling Higgs-top couplings in associated production, JHEP 04 (2014) 004 [arXiv: 1312.5736] [INSPIRE]. 
[5] F. Demartin, F. Maltoni, K. Mawatari, B. Page and M. Zaro, Higgs characterisation at NLO in QCD: CP properties of the top-quark Yukawa interaction, Eur. Phys. J. C 74 (2014) 3065 [arXiv:1407.5089] [INSPIRE].

[6] S. Khatibi and M. Mohammadi Najafabadi, Exploring the anomalous Higgs-top couplings, Phys. Rev. D 90 (2014) 074014 [arXiv:1409.6553].

[7] X.-G. He, G.-N. Li and Y.-J. Zheng, Probing Higgs boson CP properties with $t \bar{t} H$ at the LHC and the 100 TeV pp collider, Int. J. Mod. Phys. A 30 (2015) 1550156 [arXiv:1501.00012] [INSPIRE].

[8] M.R. Buckley and D. Goncalves, Boosting the direct CP measurement of the Higgs-top coupling, Phys. Rev. Lett. 116 (2016) 091801 [arXiv: 1507.07926] [INSPIRE].

[9] S. Biswas, E. Gabrielli and B. Mele, Single top and Higgs associated production as a probe of the Htt coupling sign at the LHC, JHEP 01 (2013) 088 [arXiv:1211.0499] [INSPIRE].

[10] C. Englert and E. Re, Bounding the top Yukawa coupling with Higgs-associated single-top production, Phys. Rev. D 89 (2014) 073020 [arXiv: 1402.0445] [INSPIRE].

[11] F. Demartin, F. Maltoni, K. Mawatari and M. Zaro, Higgs production in association with a single top quark at the LHC, Eur. Phys. J. C 75 (2015) 267 [arXiv:1504.00611] [INSPIRE].

[12] G. Bhattacharyya, D. Das and P.B. Pal, Modified Higgs couplings and unitarity violation, Phys. Rev. D 87 (2013) 011702 [arXiv:1212.4651] [InSPIRE].

[13] D. Choudhury, R. Islam and A. Kundu, Anomalous Higgs couplings as a window to new physics, Phys. Rev. D 88 (2013) 013014 [arXiv:1212.4652] [INSPIRE].

[14] S. Bar-Shalom, D. Atwood, G. Eilam, R.R. Mendel and A. Soni, Large tree level CP-violation in $e^{+} e^{-} \rightarrow t \bar{t} H^{0}$ in the two Higgs doublet model, Phys. Rev. D 53 (1996) 1162 [hep-ph/9508314] [INSPIRE].

[15] P.S. Bhupal Dev et al., Determining the CP properties of the Higgs boson, Phys. Rev. Lett. 100 (2008) 051801 [arXiv:0707.2878] [INSPIRE].

[16] K. Hagiwara, K. Ma and H. Yokoya, Probing CP-violation in $e^{+} e^{-}$production of the Higgs boson and toponia, JHEP 06 (2016) 048 [arXiv: 1602.00684] [INSPIRE].

[17] L. Wolfenstein, Violation of CP invariance and the possibility of very weak interactions, Phys. Rev. Lett. 13 (1964) 562 [INSPIRE].

[18] J. Alwall et al., The automated computation of tree-level and next-to-leading order differential cross sections and their matching to parton shower simulations, JHEP 07 (2014) 079 [arXiv: 1405.0301] [INSPIRE].

[19] C. Farrell and A.H. Hoang, The large Higgs energy region in Higgs associated top pair production at the linear collider, Phys. Rev. D 72 (2005) 014007 [hep-ph/0504220] [INSPIRE].

[20] C. Farrell and A.H. Hoang, Next-to-leading-logarithmic QCD corrections to the cross-section $\sigma\left(e^{+} e^{-} \rightarrow t \bar{t} H\right)$ at $500 \mathrm{GeV}$, Phys. Rev. D 74 (2006) 014008 [hep-ph/0604166] [INSPIRE].

[21] R. Yonamine et al., Measuring the top Yukawa coupling at the ILC at $\sqrt{s}=500 \mathrm{GeV}$, Phys. Rev. D 84 (2011) 014033 [arXiv: 1104.5132] [INSPIRE].

[22] Y. Sumino and H. Yokoya, Bound-state effects on kinematical distributions of top quarks at hadron colliders, JHEP 09 (2010) 034 [Erratum ibid. 1606 (2016) 037] [arXiv:1007.0075] [INSPIRE].

[23] S.-F. Ge, K. Hagiwara, N. Okamura and Y. Takaesu, Determination of mass hierarchy with medium baseline reactor neutrino experiments, JHEP 05 (2013) 131 [arXiv:1210.8141] [INSPIRE]. 
[24] K. Hagiwara and D. Zeppenfeld, Helicity amplitudes for heavy lepton production in $e^{+} e^{-}$ annihilation, Nucl. Phys. B 274 (1986) 1 [INSPIRE].

[25] K. Hagiwara, H. Murayama and I. Watanabe, Search for the Yukawa interaction in the process $e^{+} e^{-} \rightarrow t \bar{t} Z$ at TeV linear colliders, Nucl. Phys. B 367 (1991) 257 [INSPIRE].

[26] H. Murayama, I. Watanabe and K. Hagiwara, HELAS: HELicity amplitude subroutines for Feynman diagram evaluations, KEK-91-11 (1991).

[27] T. Stelzer and W.F. Long, Automatic generation of tree level helicity amplitudes, Comput. Phys. Commun. 81 (1994) 357 [hep-ph/9401258] [INSPIRE]. 